\title{
MONOTONIC DISTRIBUTIVE SEMILATTICES
}

\author{
SERGIO A. CELANI AND MA. PAULA MENCHÓN \\ CONICET and Departamento de Matemáticas, Facultad de Ciencias Exactas, Univ. \\ Nac. del Centro, Pinto 399, 7000 Tandil, Argentina
}

\begin{abstract}
In the study of algebras related to non-classical logics, (distributive) semilattices are always present in the background. For example, the algebraic semantic of the $\{\rightarrow, \wedge, \top\}$-fragment of intuitionistic logic is the variety of implicative meetsemilattices 2] 6. In this paper we introduce and study the class of distributive meet-semilattices endowed with a monotonic modal operator $m$. We study the representation theory of these algebras using the theory of canonical extensions and we give a topological duality for them. Also, we show how our new duality extends to some particular subclasses.
\end{abstract}

Distributive meet semilattices, monotonic modal logics, $D S$-spaces, modal operators.

\section{INTRODUCTION}

Boolean algebras with modal operators are the algebraic semantic of classical modal logics. Using Stone's topological representation of Boolean algebras, it is known that every Boolean algebra with a modal operator can be represented as a relational structure [5]. This representation plays an important role in the study of many extensions of normal modal logics [5] and monotone modal logics [7] [13]. Recall that monotone modal logics are a generalization of normal modal logics in which the axiom $m(\varphi \rightarrow$ $\phi) \rightarrow(m \varphi \rightarrow m \phi)$ has been weakened, leading to a monotonicity condition which can be either expressed as an axiom $(m(\varphi \wedge \phi) \rightarrow m \varphi)$, or as a rule (from $\varphi \rightarrow \phi$ derive $m \varphi \rightarrow m \phi)$. Thus, it is possible to study monotone modal logics with a language containing only the connectives $\wedge$ and $m$, or $\rightarrow$ and $m$.

Classical monotone modal logics are interpreted semantically by means of neighborhood frames [7] 13] 14. This class of structures provides a generalization of Kripke semantics. Every neighborhood frame produces a Boolean algebra endowed with a monotonic operator, called monotonic algebra. And reciprocally, every monotonic algebra defines a neighborhood frame (see [13] or [3]). Also, it is possible to consider monotone modal logics defined on non-classical logics. For example, in [16], Kojima considered

E-mail address: scelani@exa.unicen.edu.ar, mpmenchon@exa.unicen.edu.ar.

This paper has received funding from the European Union's Horizon 2020 research and innovation programme under the Marie Skłodowska-Curie grant agreement No 689176, and the support of the grant PIP $11220150100412 \mathrm{CO}$ of CONICET (Argentina). 
neighborhood semantics for intuitionistic modal logic, and he defined a neighborhood frame as a triple $\langle W, \leq, N\rangle$ where $N$ is a neighborhood function, which is a mapping from $W$ to $\mathcal{P}(\mathcal{P}(W))$ that satisfies the decreasing condition, i.e., $N(x) \supseteq N(y)$ whenever $x \leq y$ (see Definition 3.1 of [16]). Monotonic logics based on intuitionistic logic are also studied in [18].

In the study of algebras related to non-classical logics, semilattices are always present in the background. For example, the algebraic semantic of the $\{\rightarrow, \wedge, \top\}$-fragment of intuitionistic logic is the variety of implicative meet-semilattices [2] [6], and it is well known that the meet-semilattice reduct of an implicative meet-semilattice is distributive in the sense of [12] or [4]. In [12] G. Grätzer gave a topological representation for distributive semilattices using sober spaces. This representation was extended to a topological duality in [4] and [1]. The principal novelty of [4] was the characterization of meet-semilattice homomorphisms preserving top by means of certain binary relations. For implicative semilattices there exists a similar representation in [2]. The main objective of this paper is to study a full Stone style duality for distributive meet-semilattices endowed with a monotonic operator. So, most of the results given in this paper are applicable, with minor modifications, to the study of bounded distributive lattices, implicative semilattices, Heyting algebras, and Boolean algebras with monotonic operators. We note that in the particular case of Boolean algebras our duality yields the duality given in [3] and [13].

Canonical extensions were introduced by Jónsson and Tarski to study Boolean algebras with operators. The main purpose was to make it easier to identify what form the dual of an additional operation on a lattice should take. Since their seminal work, the theory of canonical extensions has been simplified and generalized [11, 8], leading to a theory widely applicable beyond the original Boolean setting. We will use canonical extension as a tool for the development of a theory of relational methods, in an algebraic way.

The paper is organized as follows. In Section 2 we recall the definitions and some basic properties of distributive semilattices and canonical extensions. We recall the topological representation and duality developed in [4] and [1]. In Section 3, we introduce a special class of saturated sets of a $D S$-space that is dual to the family of order ideals of a distributive semilattice. In Section 4 we present the class of distributive semilattices endowed with a monotonic operator, and we extend the results on representation using canonical extensions. In section 5 we consider some important applications of the duality. We show how our new duality extends to some particular subclasses.

\section{Preliminaries}

We include some elementary properties of distributive semilattices that are necessary to read this paper. For more details see [4, [6] and [1].

Let $\langle X, \leq\rangle$ be a poset. For each $Y \subseteq X$, let $[Y)=\{x \in X: \exists y \in Y(y \leq x)\}$ and $(Y]=\{x \in X: \exists y \in Y(x \leq y)\}$. If $Y=\{y\}$, then we will write $[y)$ and (y] instead of $[\{y\})$ and $(\{y\}]$, respectively. We call $Y$ an upset (resp. downset) if $Y=[Y)$ (resp. 
$Y=(Y])$. The set of all upsets of $X$ will be denoted by $\operatorname{Up}(X)$. The complement of a subset $Y \subseteq X$ will be denoted by $Y^{c}$ or $X-Y$.

Definition 1. A meet-semilattice with greatest element, a semilattice for short, is an algebra $\mathbf{A}=\langle A, \wedge, 1\rangle$ of type $(2,0)$ such that the operation $\wedge$ is idempotent, commutative, associative, and $a \wedge 1=a$ for all $a \in A$.

It is clear that for each poset $\langle X, \leq\rangle$ the structure $\langle\operatorname{Up}(X), \cap, X\rangle$ is a semilattice.

Let $\mathbf{A}$ be a semilattice. As usual, we can define a partial order on $\mathbf{A}$, called the natural order, as $a \leq b$ iff $a \wedge b=a$. It is easy to see that 1 is the greatest element of $A$. A subset $F \subseteq A$ is a filter of $\mathbf{A}$ if it is an upset, $1 \in F$ and if $a, b \in F$, then $a \wedge b \in F$. We will denote the set of all filters of $\mathbf{A}$ by $\operatorname{Fi}(\mathbf{A})$. It is easy to see that $\operatorname{Fi}(\mathbf{A})$ is closed under arbitrary intersections. The filter generated by the subset $X \subseteq A$ will be denoted by $F(X)$. If $X=\{a\}, F(\{a\})=F(a)=[a)$. We shall say that a proper filter is irreducible or prime if for any pair of filters $F_{1}, F_{2}$ such that $F=F_{1} \cap F_{2}$, it follows that $F=F_{1}$ or $F=F_{2}$.

We will denote the set of all irreducible filters of a semilattice $\mathbf{A}$ by $X(\mathbf{A})$. A subset $I \subseteq A$ is called an order ideal if it is a downset and for every $a, b \in I$ we have that there exists $c \in I$ such that $a, b \leq c$. We will denote the set of all order ideals of $\mathbf{A}$ by $\operatorname{Id}(\mathbf{A})$.

Theorem 2. 4 Let $\mathbf{A}$ be a semilattice. Let $F \in \operatorname{Fi}(\mathbf{A})$ and let $I \in \operatorname{Id}(\mathbf{A})$ such that $F \cap I=\emptyset$. Then there exists $P \in X(\mathbf{A})$ such that $F \subseteq P$ and $P \cap I=\emptyset$.

A semilattice $\mathbf{A}$ is distributive if for all $a, b, c \in A$ such that $a \wedge b \leq c$ there exist $a_{1}, b_{1} \in A$ such that $a \leq a_{1}, b \leq b_{1}$ and $c=a_{1} \wedge b_{1}$. We will denote by $\mathcal{D S}$ the class of distributive semilattices. We recall (see [1, 4]) that in a distributive semilattice $\mathbf{A}$, if $F$ is a proper filter then the following conditions are equivalent:

(1) $F$ is irreducible,

(2) for every $a, b \in A$ such that $a, b \notin F$, there exist $c \notin F$ and $f \in F$ such that $a \wedge f \leq c$ and $b \wedge f \leq c$,

(3) $A-F=F^{c}$ is an order ideal.

Let $\mathbf{A}, \mathbf{B} \in \mathcal{D S}$. A mapping $h: A \rightarrow B$ is called a semilattice homomorphism if

(1) $h(a) \wedge h(b)=h(a \wedge b)$ for every $a, b \in A$ and

(2) $h(1)=1$.

Let $\mathbf{A} \in \mathcal{D S}$. Let us consider the poset $\langle X(\mathbf{A}), \subseteq\rangle$ and the mapping $\beta_{\mathbf{A}}: A \rightarrow$ $\mathrm{Up}(X(\mathbf{A}))$ defined by $\beta_{\mathbf{A}}(a)=\{P \in X(\mathbf{A}): a \in P\}$. For convenience, we omit the subscript of $\beta_{\mathbf{A}}$, when no confusion can arise.

Theorem 3. Let $\mathbf{A} \in \mathcal{D S}$. Then $\mathbf{A}$ is isomorphic to the subalgebra $\beta[A]=\{\beta(a): a \in$ A\} of $\langle\mathrm{Up}(X(\mathbf{A})), \cap, X(\mathbf{A})\rangle$.

2.1. $\mathcal{D S}$-spaces. In this subsection we recall the duality for distributive semilattices given in [1] and [4] based on a Stone style duality and we give some definitions that we will need to extend it. 
Let $\langle X, \mathcal{T}\rangle$ be a topological space. We will denote by $\mathcal{K O}(X)$ the set of all compact and open subsets of $X$ and let $D(X)$ be the set $D(X)=\left\{U: U^{c} \in \mathcal{K} \mathcal{O}(X)\right\}$. We will denote by $\mathcal{C}(X)$ the set of all non-empty closed subsets of $X$. The closure of a subset $Y \subseteq X$ will be denoted by $\operatorname{cl}(Y)$. A subset $Y \subseteq X$ is saturated if it is an intersection of open sets. The smallest saturated set containing $Y$ is the saturation of $Y$ and will be denoted by $\operatorname{sat}(Y)$.

We recall that the specialization order of $\langle X, \mathcal{T}\rangle$ is defined by $x \preceq y$ if $x \in \operatorname{cl}(\{y\})=$ $\operatorname{cl}(y)$. It is easy to see that $\preceq$ is a reflexive and transitive relation. If $X$ is $T_{0}$ then the relation $\preceq$ is a partial order. The dual order of $\preceq$ will be denoted by $\leq$, i.e., $x \leq y$ if $y \in \operatorname{cl}(x)$. Moreover, if $X$ is $T_{0}$ then $\operatorname{cl}(x)=[x)$, sat $(Y)=(Y]$, and every open (resp. closed) subset is a downset (resp. upset) respect to $\leq$.

Recall that a non-empty subset $Y \subseteq X$ of a topological space $\langle X, \mathcal{T}\rangle$ is irreducible if $Y \subseteq Z \cup W$ for any closed subsets $Z$ and $W$, implies $Y \subseteq Z$ or $Y \subseteq W$. A topological space $\langle X, \mathcal{T}\rangle$ is sober if for every irreducible closed set $Y \subseteq X$, there exists a unique $x \in X$ such that $\operatorname{cl}(x)=Y$. Each sober space is $T_{0}$. The following definition is equivalent to the definition given by G. Grätzer in [12].

Definition 4. [1] A $D S$-space is a topological space $\langle X, \mathcal{T}\rangle$ such that:

(1) The set of all compact and open subsets $\mathcal{K O}(X)$ forms a basis for the topology $\mathcal{T}$ on $X$.

(2) $\langle X, \mathcal{T}\rangle$ is sober.

If $\langle X, \mathcal{T}\rangle$ is a $D S$-space, then $\langle D(X), \cap, X\rangle$ is a distributive semilattice (see [12]).

Let $\langle X, \leq\rangle$ be a poset. Recall that a subset $K \subseteq X$ is called dually directed if for any $x, y \in K$ there exists $z \in K$ such that $z \leq x$ and $z \leq y$. A subset $K \subseteq X$ is called directed if for any $x, y \in K$ there exists $z \in K$ such that $x \leq z$ and $y \leq z$.

Theorem 5. Let $\langle X, \mathcal{T}\rangle$ be a topological space with basis $\mathcal{K}$ of open and compact subsets for $\mathcal{T}$. Then, the following conditions are equivalent:

(1) $\langle X, \mathcal{T}\rangle$ is sober

(2) $\langle X, \mathcal{T}\rangle$ is $T_{0}$ and $\bigcap\{U: U \in \mathcal{L}\} \cap Y \neq \emptyset$ for each closed subset $Y$ and for any dually directed subset $\mathcal{L} \subseteq \mathcal{K}$ such that $Y \cap U \neq \emptyset$ for all $U \in \mathcal{L}$.

Let $\mathbf{A} \in \mathcal{D S}$. Consider $\mathcal{K}_{\mathbf{A}}=\left\{\beta(a)^{c}: a \in A\right\}$ and let $\mathcal{T}_{\mathbf{A}}$ be the topology generated by the basis $\mathcal{K}_{\mathbf{A}}$. Then, $\left\langle X(\mathbf{A}), \mathcal{T}_{\mathbf{A}}\right\rangle$ is a $D S$-space, called the dual space of $\mathbf{A}$ (see [4] and [1]). Recall that $Q \in \operatorname{cl}(P)$ iff $P \subseteq Q$, i.e., the specialization dual order of $\left\langle X(\mathbf{A}), \mathcal{T}_{\mathbf{A}}\right\rangle$ is the inclusion relation $\subseteq$. Also, recall that the lattices $\operatorname{Fi}(\mathbf{A})$ and $\mathcal{C}(X(\mathbf{A}))$ are dually isomorphic under the maps $F \mapsto \hat{F}$, where $\hat{F}=\{P \in X(\mathbf{A}): F \subseteq P\}=\bigcap\{\beta(a)$ : $a \in F\}$ for each $F \in \operatorname{Fi}(\mathbf{A})$ and $Y \mapsto F_{Y}$, where $F_{Y}=\{a \in A: Y \subseteq \beta(a)\}$ for each $Y \in \mathcal{C}(X(\mathbf{A}))$.

2.2. Canonical extension. Here we will give the basic definitions of the theory of canonical extensions focused on (distributive) meet semilattices. The following is an adaptation of the definition given in [8] for posets. This definition agrees with the 
definition of canonical extensions for bounded distributive lattices and Boolean algebras [11, 15].

Definition 6. Let $\mathbf{A}$ be a semilattice. A completion of $\mathbf{A}$ is a semilattice embedding $e: A \rightarrow X$ where $X$ is a complete lattice. From now on, we will suppress $e$ and call $X$ a completion of $\mathbf{A}$ and assume that $\mathbf{A}$ is a subalgebra of $X$.

Definition 7. Let $\mathbf{A}$ be a semilattice. Given a completion $X$ of $\mathbf{A}$, an element of $X$ is called closed provided it is the infimum in $X$ of some filter $F$ of $\mathbf{A}$. We denote the set of all closed elements of $X$ by $K(X)$. Dually, an element of $X$ is called open provided it is the supremum in $X$ of some order ideal $I$ of $\mathbf{A}$. We denote the set of all open elements of $X$ by $O(X)$. A completion $X$ of $\mathbf{A}$ is said to be dense provided each element of $X$ is both the supremum of all the closed elements below it and the infimum of all the open elements above it. A completion $X$ of $\mathbf{A}$ is said to be compact provided that whenever $D$ is a non-empty dually directed subset of $A, U$ is a non-empty directed subset of $A$, and $\bigwedge_{L} D \leq \bigvee_{L} U$, then there exist $x \in D$ and $y \in U$ such that $x \leq y$.

Definition 8. Let $\mathbf{A}$ be a semilattice. A canonical extension of $\mathbf{A}$ is a dense and compact completion of $\mathbf{A}$.

Theorem 9. Let $\mathbf{A}$ be a semilattice, then $\mathbf{A}$ has a canonical extension and it is unique up to an isomorphism that fixes $\mathbf{A}$.

Lemma 10. Let us consider a distributive semilattice with greatest element $\mathbf{A}=\langle A, \wedge, 1\rangle$. $\langle\mathrm{Up}(X(\mathbf{A})), \cap, \cup, X(\mathbf{A}), \emptyset\rangle$ is a canonical extension of $\mathbf{A}$, where $A \cong \beta[A] \subseteq \mathrm{Up}(X(\mathbf{A}))$. We will call it 'the' canonical extension.

\section{IdEALS AND SATURATED SUbSETS}

In this section we present a particular family of saturated sets in a $D S$-space, dual to the family of order ideals of a semilattice.

Definition 11. Let $\langle X, \mathcal{T}\rangle$ be a $D S$-space. $Z \subseteq X$ is a special basic saturated subset if $Z=\bigcap\{W: W \in \mathcal{L}\}$ for some dually directed family $\mathcal{L} \subseteq \mathcal{K O}(X)$.

We denote by $\mathcal{S}(X)$ the set of all special basic saturated subsets of a $D S$-space $\langle X, \mathcal{T}\rangle$. Note that every special basic saturated subset is a saturated set.

Let $\mathbf{A} \in \mathcal{D} \mathcal{S}$. Let $I \in \operatorname{Id}(\mathbf{A})$. We consider the following subset of $X(\mathbf{A})$ :

$$
\alpha(I)=\bigcap\left\{\beta(a)^{c}: a \in I\right\}=\{P \in X(\mathbf{A}): I \cap P=\emptyset\} .
$$

It is clear that $\alpha(I)$ is a special basic saturated set of $\left\langle X(\mathbf{A}), \mathcal{T}_{\mathbf{A}}\right\rangle$. Let $Z \subseteq X(\mathbf{A})$ be a special basic saturated set of $X(\mathbf{A})$. Consider the subset

$$
I_{\mathbf{A}}(Z)=\{a \in A: \beta(a) \cap Z=\emptyset\} .
$$

It is easy to see that $I_{\mathbf{A}}(Z)$ is a downset of $\mathbf{A}$.

Remark 12. The special basic saturated subsets of a $D S$-space $\langle X, \mathcal{T}\rangle$ are precisely the compact saturated subsets of the topology. 
Given two posets $\left\langle X, \leq_{X}\right\rangle$ and $\left\langle Y, \leq_{Y}\right\rangle$, a surjective order-isomorphism from $\left\langle X, \leq_{X}\right\rangle$ to $\left\langle Y, \leq_{Y}\right\rangle$ is a surjective function $f: X \rightarrow Y$ with the property that for every $x$ and $y$ in $X, x \leq_{X} y$ if and only if $f(x) \leq_{Y} f(y)$. We say that the posets $\left\langle X, \leq_{X}\right\rangle$ and $\left\langle Y, \leq_{Y}\right\rangle$ are isomorphic if there exists a surjective order-isomorphism $f: X \rightarrow Y$.

In the following result we prove that order ideals are in bijective correspondece with the family of basic saturated subsets of $X(\mathbf{A})$.

Theorem 13. Let $\mathbf{A} \in \mathcal{D S}$. Then the posets $\langle\operatorname{Id}(\mathbf{A}), \subseteq\rangle$ and $\langle\mathcal{S}(X(\mathbf{A})), \subseteq\rangle$ are dually isomorphic.

Proof. Let $Z \subseteq X(\mathbf{A})$ be a special basic saturated subset of $X(\mathbf{A})$. We prove that $I_{\mathbf{A}}(Z)$ is an order ideal of $\mathbf{A}$ and $Z=\alpha\left(I_{\mathbf{A}}(Z)\right)$. Moreover, if $I$ is any order ideal of $\mathbf{A}$, then we prove that $I=I_{\mathbf{A}}(\alpha(I))$.

It is clear that $I_{\mathbf{A}}(Z)$ is a downset of $\mathbf{A}$. Let $a, b \in I_{\mathbf{A}}(Z)$. So, we have that $Z \cap\left(\beta_{\mathbf{A}}(a) \cup\right.$ $\left.\beta_{\mathbf{A}}(b)\right)=\emptyset$. Since $Z=\bigcap\left\{\beta(a)^{c}: \beta(a)^{c} \in \mathcal{L}\right\}$ for some dually directed family $\mathcal{L} \subseteq \mathcal{K}_{\mathbf{A}}$ and $\beta(a) \cup \beta(b)$ is a closed subset, there exists $\beta_{\mathbf{A}}(c)^{c} \in \mathcal{L}$ such that $\beta(c)^{c} \cap(\beta(a) \cup \beta(b))=\emptyset$. Thus, $\beta(a) \cup \beta(b) \subseteq \beta(c)$ and $Z \cap \beta_{\mathbf{A}}(c)=\emptyset$, i.e., $a, b \leq c$ and $c \in I_{\mathbf{A}}(Z)$. Therefore, $I_{\mathbf{A}}(Z)$ is an order ideal of $\mathbf{A}$ and we have that $\alpha\left(I_{\mathbf{A}}(Z)\right)=\bigcap\left\{\beta(a)^{c}: Z \subseteq \beta(a)^{c}\right\} \subseteq$ $\bigcap\left\{\beta(a)^{c}: \beta(a)^{c} \in \mathcal{L}\right\}=Z$. The other inclusion is immediate.

Now, let $I$ be an order ideal. Let $b \in I_{\mathbf{A}}(\alpha(I))$. Then $\beta(b) \cap \alpha(I)=\beta(b) \cap \bigcap\left\{\beta(a)^{c}\right.$ : $a \in I\}=\emptyset$. Since $\beta_{\mathbf{A}}(b)$ is a closed subset, and the family $\left\{\beta(a)^{c}: a \in I\right\}$ is dually directed, we get that there exists $a \in I$ such that $\beta(b) \subseteq \beta(a)$. So, $b \leq a$, and as $I$ is a downset, we have that $b \in I$. The other inclusion is immediate.

Thus, we have a surjective function $\alpha: \operatorname{Id}(\mathbf{A}) \rightarrow \mathcal{S}(X(\mathbf{A}))$ with inverse function $I_{\mathbf{A}}: \mathcal{S}(X(\mathbf{A})) \rightarrow \operatorname{Id}(\mathbf{A})$. We prove that $\alpha$ is a dual order-isomorphism. Let $I_{1}$ and $I_{2}$ be two ideals of $\mathbf{A}$. Assume that $I_{1} \subseteq I_{2}$. Let $P \in \alpha\left(I_{2}\right)$. Then, $P \cap I_{2}=\emptyset$. It follows that $P \cap I_{1}=\emptyset$, i.e., $P \in \alpha\left(I_{1}\right)$.

Assume that $\alpha\left(I_{1}\right) \subseteq \alpha\left(I_{2}\right)$. Let $a \in I_{2}$ and suppose that $a \notin I_{1}$. Then $I_{1} \cap[a)=\emptyset$, so there exists $P \in X(\mathbf{A})$ such that $[a) \subseteq P$ and $P \cap I_{1}=\emptyset$. It follows that $P \in \alpha\left(I_{1}\right)$ but $P \notin \alpha\left(I_{2}\right)$ which is a contradiction. Therefore, $a \in I_{1}$.

Remark 14. We note that for any $a \in A, \alpha((a])=\beta(a)^{c}$.

For simplicity we will write $\alpha(a)$ instead of $\alpha((a])$.

Proposition 15. Let $\mathbf{A} \in \mathcal{D S}$, let $Y \in \mathcal{C}(X(\mathbf{A}))$ and $Z \in \mathcal{S}(X(\mathbf{A}))$. Then,

$$
F_{Y} \cap I_{\mathbf{A}}(Z)=\emptyset \text { iff } Y \cap Z \neq \emptyset \text {. }
$$

Proof. Suppose that $F_{Y} \cap I_{\mathbf{A}}(Z)=\emptyset$. Then, there exists $P \in X(\mathbf{A})$ such that $F_{Y} \subseteq P$ and $P \cap I_{\mathbf{A}}(Z)=\emptyset$, i.e., $P \in Y$ and $P \in Z$. Thus, $Y \cap Z \neq \emptyset$. The rest of proof is straightforward.

Now, we are able to identify the topological structures that are the closed and open elements of a canonical extension of a distributive semilattice.

Lemma 16. Let $\mathbf{A}$ be a distributive semilattice. Let us consider the canonical extension $\langle\operatorname{Up}(X(\mathbf{A})), \cap, \cup, X(\mathbf{A}), \emptyset\rangle$ and the $D S$-space $\left\langle X(\mathbf{A}), \mathcal{T}_{\mathbf{A}}\right\rangle$. Then, $K(\operatorname{Up}(X(\mathbf{A})))=$ 
$\mathcal{C}(X(\mathbf{A}))$ and $O(\operatorname{Up}(X(\mathbf{A})))=\left\{Z^{c}: Z \in \mathcal{S}(X(\mathbf{A}))\right\}$, i.e., the closed elements of the canonical extension are exactly the closed sets of the topology and the open elements of the canonical extension are the complements of the special saturated sets of the topology.

Remark 17. Given a complete lattice $C$, we denote the set of completely join prime elements by $J^{\infty}(C)$ and the set of completely meet prime elements by $M^{\infty}(C)$.

Every element of $\operatorname{Up}(X(\mathbf{A}))$ is a join of completely join prime elements and a meet of completely meet prime elements, where $J^{\infty}(\operatorname{Up}(X(\mathbf{A})))=\{\hat{P}=[P): P \in X(\mathbf{A})\}$ and $M^{\infty}(\mathrm{Up}(X(\mathbf{A})))=\left\{\alpha\left(P^{c}\right)^{c}=(P]^{c}: P \in X(\mathbf{A})\right\}$.

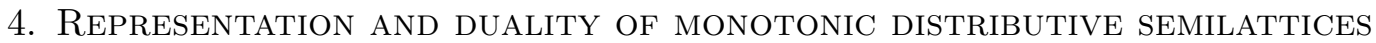

Definition 18. Let $\mathbf{A}=\langle A, \wedge, 1\rangle$ be a distributive semilattice. A monotonic operator is an operator $m: A \rightarrow A$ that satisfies the following condition

$$
\text { If } a \leq b \text {, then } m a \leq m b \text { for all } a, b \in A \text {. }
$$

The following result is immediate.

Proposition 19. Let $\mathbf{A} \in \mathcal{D S}$ and let $m: A \rightarrow A$ be a unary function. Then the following conditions are equivalent:

(1) For all $a, b \in A$, if $a \leq b$ then $m a \leq m b$,

(2) $m(a \wedge b) \leq m a \wedge m b$ for all $a, b \in A$.

Definition 20. Let $\mathbf{A} \in \mathcal{D S}$. The pair $\langle\mathbf{A}, m\rangle$ such that $m$ is a monotonic operator defined on $\mathbf{A}$ is called a monotonic distributive semilattice.

The class of all monotonic distributive semilattices will be denoted by $\mathcal{M D S}$.

Let $\langle\mathbf{A}, m\rangle,\langle\mathbf{B}, m\rangle \in \mathcal{M D S}$. We say that a homomorphism $h: A \rightarrow B$ is a homomorphism of monotonic distributive semilattices if $h$ commutes with $m$, i.e., if $h(m a)=$ $m h(a)$ for all $a \in A$. We denote by $\mathcal{M D S H}$ the category of monotonic distributive semilattices and monotonic distributive semilattice homomorphisms.

We will give two examples of monotonic distributive semilattices constructed from certain relational systems. We shall use these examples in the theory of representation and topological duality for monotonic distributive semilattices.

Let $X$ be a set. A multirelation on $X$ is a subset of the Cartesian product $X \times \mathcal{P}(X)$, that is, a set of ordered pairs $(x, Y)$ where $x \in X$ and $Y \subseteq X$ [9] [17]. We recall that in classical monotone modal logic a neighborhood frame is a pair $\langle X, R\rangle$ where $X$ is a set and $R \subseteq X \times \mathcal{P}(X)$, i.e., $R$ is a multirelation (see [7] [13]). Now we give a generalization of this notion.

Definition 21. An $S$-neighborhood frame is a triple $\langle X, \leq, R\rangle$ where $\langle X, \leq\rangle$ is a poset and $R$ is a subset of $X \times \mathcal{P}(X)$ such that if $x \leq y$, then $R(y) \subseteq R(x)$ for all $x, y \in X$. For each $U \in \operatorname{Up}(X)$ we define the set

$$
m_{R}(U)=\{x \in X: \forall Z \in R(x)(Z \cap U \neq \emptyset)\} .
$$


A $C$-neighborhood frame is a triple $\langle X, \leq, G\rangle$ where $\langle X, \leq\rangle$ is a poset and $G$ is a subset of $X \times \mathcal{P}(X)$ such that if $x \leq y$, then $G(x) \subseteq G(y)$ for all $x, y \in X$. For each $U \in \operatorname{Up}(X)$ we define the set

$$
\mathbf{m}_{G}(U)=\{x \in X: \exists Y \in G(x)(Y \subseteq U)\} .
$$

Lemma 22. Let $\langle X, \leq, R\rangle$ be an $S$-neighborhood frame and $\langle X, \leq, G\rangle$ be a $C$-neighborhood frame. Then $\left\langle\mathrm{Up}(X), \cap, m_{R}, X\right\rangle$ and $\left\langle\mathrm{Up}(X), \cap, \mathbf{m}_{G}, X\right\rangle$ are monotonic distributive semilattices.

We will represent the monotonic operator $m$ on a distributive semilattice $\mathbf{A}$ as a multirelation on the dual space of $\mathbf{A}$, where the canonical extension offers an advantageous point of view. We consider two different ways of extending maps that agree with the ones given in $[8$ for posets, bounded distributive lattices and Boolean algebras.

Definition 23. Let $\mathbf{A}$ be a distributive semilattice. Given a monotonic operation $m: A \rightarrow A$, we define the maps

$$
m^{\sigma}, m^{\pi}: \operatorname{Up}(X(\mathbf{A})) \rightarrow \mathrm{Up}(X(\mathbf{A}))
$$

by

and

$$
m^{\sigma}(X)=\bigcup\{\bigcap\{\beta(m a): Y \subseteq \beta(a)\}: X \supseteq Y \in \mathcal{C}(X(\mathbf{A}))\}
$$

$$
m^{\pi}(X)=\bigcap\left\{\bigcup\left\{\beta(m a): Z \subseteq \beta(a)^{c}\right\}: X^{c} \supseteq Z \in \mathcal{S}(X(\mathbf{A}))\right\} .
$$

The two extensions of a map $m$ shown above are not always equal. Whether we want to extend a particular additional operation using the $\sigma$-or the $\pi$-extension depends on the properties of the particular operation to be extended. The following lemma is a consequence of Lemma 3.4 of [8].

Lemma 24. Let $\langle\mathbf{A}, m\rangle \in \mathcal{M D S}$. The maps $m^{\sigma}, m^{\pi}$ are monotonic extensions of $m$, i.e., $\left\langle\operatorname{Up}(X(\mathbf{A})), m^{\sigma}\right\rangle,\left\langle\operatorname{Up}(X(\mathbf{A})), m^{\pi}\right\rangle \in \mathcal{M D S}$ and $m^{\sigma}(\beta(a))=m^{\pi}(\beta(a))=\beta(m a)$ for all $a \in A$. In addition, $m^{\sigma} \leq m^{\pi}$ with equality holding on $K(\operatorname{Up}(X(\mathbf{A}))) \cup O(\operatorname{Up}(X(\mathbf{A})))$. For $X \in \mathrm{Up}(X(\mathbf{A})), Y \in \mathcal{C}(X(\mathbf{A}))$ and $Z \in \mathcal{S}(X(\mathbf{A}))$

$$
\begin{aligned}
& m^{\sigma}(X)=\bigcup\left\{m^{\sigma}(Y): X \supseteq Y \in \mathcal{C}(X(\mathbf{A}))\right\}, \\
& m^{\sigma}(Y)=\bigcap\{\beta(m a): Y \subseteq \beta(a)\} \\
& m^{\pi}(X)=\bigcap\left\{m^{\pi}\left(Z^{c}\right): X^{c} \supseteq Z \in \mathcal{S}(X(\mathbf{A}))\right\}, \\
& m^{\pi}\left(Z^{c}\right)=\bigcup\left\{\beta(m a): Z \subseteq \beta(a)^{c}\right\} .
\end{aligned}
$$

So, $m^{\sigma}$ and $m^{\pi}$ send closed sets to closed sets and complements of special saturated sets to complements of special saturated sets.

Now we show how, using the $\sigma$-extension and the $\pi$-extension, it is possible to define two multirelations on the dual space of $\mathbf{A}$.

Let $\langle\mathbf{A}, m\rangle \in \mathcal{M D S}$. Note that by definition of $m^{\pi}$, for every $Z \in \mathcal{S}(X(\mathbf{A}))$ we have:

$$
\begin{aligned}
P \in m^{\pi}\left(Z^{c}\right) & \Leftrightarrow \exists a \in A \text { such that } P \in \beta(m a) \text { and } Z \subseteq \beta(a)^{c} \\
& \Leftrightarrow \exists a \in A \text { such that } m a \in P \text { and } a \in I_{\mathbf{A}}(Z) \\
& \Leftrightarrow m^{-1}(P) \cap I_{\mathbf{A}}(Z) \neq \emptyset .
\end{aligned}
$$


So, for every $X \in \operatorname{Up}(X(\mathbf{A}))$ we get:

$P \in m^{\pi}(X) \Leftrightarrow \forall Z \in \mathcal{S}(X(\mathbf{A}))$ such that $Z \subseteq X^{c}$, we have $P \in m^{\pi}\left(Z^{c}\right)$

$$
\Leftrightarrow \forall Z \in \mathcal{S}(X(\mathbf{A})) \text { such that } Z \subseteq X^{c} \text {, we have } m^{-1}(P) \cap I_{\mathbf{A}}(Z) \neq \emptyset \text {. }
$$

We define the relation

$$
R_{m} \subseteq X(\mathbf{A}) \times \mathcal{S}(X(\mathbf{A}))
$$

by

$$
(P, Z) \in R_{m} \text { iff } m^{-1}(P) \cap I_{\mathbf{A}}(Z)=\emptyset .
$$

Consequently, the operation $m^{\pi}$ on $\operatorname{Up}(X(\mathbf{A}))$ can be defined in terms of the relation $R_{m}$ as:

$$
P \in m^{\pi}(X) \text { iff } \forall Z \in R_{m}(P)[Z \cap X \neq \emptyset] .
$$

On the other hand, we can define another multirelation using the operation $m^{\sigma}$. Note that for each $Y \in \mathcal{C}(X(\mathbf{A}))$ we have:

$$
\begin{aligned}
P \in m^{\sigma}(Y) & \Leftrightarrow \forall a \in A \text { such that } Y \subseteq \beta(a) \text { we get } P \in \beta(m a) \\
& \Leftrightarrow \forall a \in A \text { such that } a \in F_{Y} \text { we get } m a \in P \\
& \Leftrightarrow F_{Y} \subseteq m^{-1}(P) .
\end{aligned}
$$

So, for each $X \in \operatorname{Up}(X(\mathbf{A}))$ we obtain:

$$
\begin{aligned}
P \in m^{\sigma}(X) & \Leftrightarrow \exists Y \in \mathcal{C}(X(\mathbf{A})) \text { such that } Y \subseteq X \text { and } P \in m^{\sigma}(Y) \\
& \Leftrightarrow \exists Y \in \mathcal{C}(X(\mathbf{A})) \text { such that } Y \subseteq X \text { and } P \in m^{\sigma}(Y) \\
& \Leftrightarrow \exists Y \in \mathcal{C}(X(\mathbf{A})) \text { such that } Y \subseteq X \text { and } F_{Y} \subseteq m^{-1}(P) .
\end{aligned}
$$

Thus, we can define another relation $G_{m} \subseteq X(\mathbf{A}) \times \mathcal{C}(X(\mathbf{A}))$ as

$$
(P, Y) \in G_{m} \text { iff } F_{Y} \subseteq m^{-1}(P) .
$$

Consequently, the operation $m^{\sigma}$ on $\operatorname{Up}(X(\mathbf{A}))$ can be defined in terms of the relation $G_{m}$ as:

$$
P \in m^{\sigma}(X) \text { iff } \exists Y \in G_{m}(P)[Y \subseteq X] .
$$

Remark 25. Let $\langle\mathbf{A}, m\rangle \in M D S$. Note that $\left\langle X(\mathbf{A}), \subseteq, R_{m}\right\rangle$ and $\left\langle X(\mathbf{A}), \subseteq, G_{m}\right\rangle$ are an S-monotonic and a C-monotonic frame, respectively, where $m_{R_{m}}=m^{\pi}$ and $\mathbf{m}_{G_{m}}=m^{\sigma}$.

Now, we are able to define the dual spaces of monotonic distributive semilattices. Depending on the way we define the relation on the dual space, there are two possible constructions of relational systems. However, we will show that both systems are interdefinible. For each monotonic operator, we can choose to work with either of them based on its behavior. In the next section we will see how some additional conditions affect the relations associated.

Let $\langle X, \mathcal{K}\rangle$ be a $D S$-space. For each $U \in D(X)$ we define the subsets $L_{U}$ and $D_{U}$ of $\mathcal{P}(\mathcal{S}(X))$ and $\mathcal{P}(\mathcal{C}(X))$ as follows:

$$
L_{U}=\{Z \in \mathcal{S}(X): Z \cap U \neq \emptyset\}
$$


and

$$
D_{U}=\{Y \in \mathcal{C}(X): Y \subseteq U\} .
$$

Definition 26. An $\mathcal{S}$-monotonic $D S$-space is a structure $\langle X, \mathcal{T}, R\rangle$, where $\langle X, \mathcal{T}\rangle$ is a $D S$-space, and $R \subseteq X \times \mathcal{S}(X)$ is a multirelation such that

(1) $m_{R}(U)=\{x \in X: \forall Z \in R(x)[Z \cap U \neq \emptyset]\} \in D(X)$, for all $U \in D(X)$ and

(2) $R(x)=\bigcap\left\{L_{U}: U \in D(X)\right.$ and $\left.x \in m_{R}(U)\right\}$, for all $x \in X$.

We can also give an analogous definition of $\mathcal{C}$-monotonic $D S$-space as a structure $\langle X, \mathcal{T}, G\rangle$, where $\langle X, \mathcal{T}\rangle$ is a $D S$-space and $G \subseteq X \times \mathcal{C}(X)$ is a multirelation such that

(3) $\mathbf{m}_{G}(U)=\{x \in X: \exists Y \in G(x)[Y \subseteq U]\} \in D(X)$ for all $U \in D(X)$, and

(4) $G(x)=\bigcap\left\{\left(D_{U}\right)^{c}: U \in D(X)\right.$ and $\left.x \in \mathbf{m}_{G}(U)^{c}\right\}$ for all $x \in X$.

Lemma 27. Let $\langle X, \mathcal{T}, R\rangle$ and $\langle X, \mathcal{T}, G\rangle$ be an $\mathcal{S}$-monotonic $D S$-space and a $\mathcal{C}$-monotonic $D S$-space respectively. Then,

(1) $R(y) \subseteq R(x)$ for all $x, y \in X$ such that $x \leq y$ and

(2) $G(x) \subseteq G(y)$ for all $x, y \in X$ such that $x \leq y$.

Proof. 1. Suppose that $x \leq y$ and let $Z \in R(y)$. Let $U \in D(X)$ such that $x \in m_{R}(U)$. By (1) of Definition 26, $m_{R}(U)$ is an upset, then $y \in m_{R}(U)$. By (2) of Definition 26 we have that $Z \cap U \neq \emptyset$. Then, $Z \in \bigcap\left\{L_{U}: U \in D(X)\right.$ and $\left.x \in m_{R}(U)\right\}=R(x)$.

2. Suppose that $x \leq y$. Let $Y \in G(x)$. Let $U \in D(X)$ such that $y \in \mathbf{m}_{G}(U)^{c}$. By (3) of Definition 26, $\mathbf{m}_{G}(U)^{c}$ is a downset, then $x \in \mathbf{m}_{G}(U)^{c}$. By (4) of Definition 26 we have that $Y \cap U^{c} \neq \emptyset$. Then, $Y \in \bigcap\left\{\left(D_{U}\right)^{c}: U \in D(X)\right.$ and $\left.y \in \mathbf{m}_{G}(U)^{c}\right\}=G(x)$.

As a corollary we have that $\langle X, \leq, R\rangle$ is an S-neighborhood frame and $\langle X, \leq, G\rangle$ is a C-neighborhood frame. From Definition 21, we get that the algebras $\left\langle\operatorname{Up}(X), m_{R}\right\rangle$ and $\left\langle\mathrm{Up}(X), \mathbf{m}_{G}\right\rangle$, considering the operators defined by 4.1 and 4.2 , are monotonic distributive semilattices. In consequence, by (1) and (3) of Definition $26,\left\langle D(X), m_{R}\right\rangle$ and $\left\langle D(X), \mathbf{m}_{G}\right\rangle$ are monotonic distributive semilattices considering the operators restricted to $D(X)$.

Now, we will see how we get a kind of space from the other.

Definition 28. Let $\langle X, \mathcal{T}\rangle$ be a $D S$-space. Let $\phi_{X}: \mathcal{P}(\mathcal{S}(X)) \rightarrow \mathcal{P}(\mathcal{C}(X))$ be the function defined by

$$
\phi_{X}(S)=\{Y \in \mathcal{C}(X): \forall Z \in S[Y \cap Z \neq \emptyset]\}
$$

and let $\psi_{X}: \mathcal{P}(\mathcal{C}(X)) \rightarrow \mathcal{P}(\mathcal{S}(X))$ be the function defined by

$$
\psi_{X}(C)=\{Z \in \mathcal{S}(X): \forall Y \in C[Y \cap Z \neq \emptyset]\} .
$$

It is easy to see that

$$
C \subseteq \phi_{X}(S) \text { iff } S \subseteq \psi_{X}(C),
$$

for all $S \subseteq \mathcal{S}(X)$ and $C \subseteq \mathcal{C}(X)$. It follows that the pair $\left(\phi_{X}, \psi_{X}\right)$ is a Galois connection. 
Proposition 29. (1) Given an $\mathcal{S}$-monotonic $D S$-space $\langle X, \mathcal{T}, R\rangle$, the relation $G_{R} \subseteq$ $X \times \mathcal{C}(X)$ defined as

$$
(x, Y) \in G_{R} \text { iff } Y \in \phi_{X}(R(x))
$$

is such that $\left\langle X, \mathcal{T}, G_{R}\right\rangle$ is a $\mathcal{C}$-monotonic DS-space and $m_{R}(U)=\mathbf{m}_{G_{R}}(U)$ for all $U \in D(X)$.

(2) Given a $\mathcal{C}$-monotonic $D S$-space $\langle X, \mathcal{T}, G\rangle$, the relation $R_{G} \subseteq X \times \mathcal{S}(X)$ defined as

$$
(x, Z) \in R_{G} \text { iff } Z \in \psi_{X}(G(x))
$$

is such that $\left\langle X, \mathcal{T}, R_{G}\right\rangle$ is a $\mathcal{S}$-monotonic $D S$-space and $\mathbf{m}_{G}(U)=m_{R_{G}}(U)$ for all $U \in D(X)$.

Proof. 1. Let $\langle X, \mathcal{T}, R\rangle$ be an $\mathcal{S}$-monotonic $D S$-space. We will see that $m_{R}(U)=$ $\mathbf{m}_{G_{R}}(U)$ for all $U \in D(X)$. Let $U \in D(X)$ and $x \in m_{R}(U)$. Then, for all $Z \in R(x)$ we have that $Z \cap U \neq \emptyset$ and since $U \in \mathcal{C}(X), U \in G_{R}(x)$. From $U \subseteq U$, we get that $x \in \mathbf{m}_{G_{R}}(U)$. Now, suppose that $x \in \mathbf{m}_{G_{R}}(U)$. So, there exists $Y \in G_{R}(x)$ such that $Y \subseteq U$ and since for all $Z \in R(x)$ we have that $Y \cap Z \neq \emptyset$, then $Z \cap U \neq \emptyset$ for all $Z \in R(x)$ and thus $x \in m_{R}(U)$.

We have proved that $m_{R}(U)=\mathbf{m}_{G_{R}}(U)$ and since $\langle X, T, R\rangle$ is an $\mathcal{S}$-monotonic $D S$ space, $\mathbf{m}_{G_{R}}(U) \in D(X)$.

Now, we will see that $G_{R}(x)=\bigcap\left\{\left(D_{U}\right)^{c}: U \in D(X)\right.$ and $\left.x \in \mathbf{m}_{G_{R}}(U)^{c}\right\}$ for all $x \in X$. Let $x \in X$. It is easy to prove the inclusion $G_{R}(x) \subseteq \bigcap\left\{\left(D_{U}\right)^{c}: U \in\right.$ $D(X)$ and $\left.x \in \mathbf{m}_{G_{R}}(U)^{c}\right\}$. To prove the other inclusion, let $Y \in \bigcap\left\{\left(D_{U}\right)^{c}: U \in\right.$ $D(X)$ and $\left.x \in \mathbf{m}_{G_{R}}(U)^{c}\right\}$ and suppose that $Y \notin G_{R}(x)$. So, there exists $Z \in R(x)$ such that $Z \cap Y=\emptyset$. Since $Z \in \mathcal{S}(X)$ and $Y \in \mathcal{C}(X)$, there exists $U \in D(X)$ such that $Z \subseteq U^{c}$ and $Y \cap U^{c}=\emptyset$. Then $Z \cap U=\emptyset, x \notin m_{R}(U)=\mathbf{m}_{G_{R}}(U)$ and $Y \subseteq U$, i.e., $Y \in D_{U}$, which is a contradiction.

2. Let $\langle X, \mathcal{T}, G\rangle$ be a $\mathcal{C}$-monotonic $D S$-space. We will see that $\mathbf{m}_{G}(U)=m_{R_{G}}(U)$ for all $U \in D(X)$. Let $U \in D(X)$ and $x \in m_{R_{G}}(U)$. Suppose that $x \notin \mathbf{m}_{G}(U)$. Then, for all $Y \in G(x), Y \cap U^{c} \neq \emptyset$. So, $U^{c} \in R_{G}(x)$ which contradicts the fact that $x \in m_{R_{G}}(U)$. Now, suppose that $x \in \mathbf{m}_{G}(U)$. Then there exists $Y \in G(x)$ such that $Y \subseteq U$. Let $Z \in R_{G}(x)$. So, we have that $Y \cap Z \neq \emptyset$, then $Z \cap U \neq \emptyset$. Thus $x \in m_{R_{G}}(U)$.

We have proved that $\mathbf{m}_{G}(U)=m_{R_{G}}(U)$ and since $\langle X, \mathcal{T}, G\rangle$ is a $\mathcal{C}$-monotonic $D S$ space, $m_{R_{G}}(U) \in D(X)$.

Now, we will see that $R_{G}(x)=\bigcap\left\{L_{U}: U \in D(X)\right.$ such that $\left.x \in m_{R_{G}}(U)\right\}$ for all $x \in X$. Let $x \in X$. The proof of the inclusion $R_{G} \subseteq \bigcap\left\{L_{U}: U \in D(X)\right.$ such that $x \in$ $\left.m_{R_{G}}(U)\right\}$ is easy. Let $Z \in \bigcap\left\{L_{U}: U \in D(X)\right.$ such that $\left.x \in m_{R_{G}}(U)\right\}$ and suppose that $Z \notin R_{G}(x)$. So, there exists $Y \in G(x)$ such that $Z \cap Y=\emptyset$. Since $Z \in \mathcal{S}(X)$ and $Y \in \mathcal{C}(X)$, there exists $U \in D(X)$ such that $Z \subseteq U^{c}$ and $Y \cap U^{c}=\emptyset$. Then, $Y \subseteq U$, $x \in \mathbf{m}_{G}(U)=m_{R_{G}}(U)$ and $Z \cap U=\emptyset$, i.e., $Z \notin L_{U}$, which is a contradiction.

Proposition 30. Let $\langle\mathbf{A}, m\rangle \in \mathcal{M D S}$. Then $\left\langle X(\mathbf{A}), \mathcal{T}_{\mathbf{A}}, R_{m}\right\rangle$ is an $\mathcal{S}$-monotonic $D S$ space and $\left\langle X(\mathbf{A}), \mathcal{T}_{\mathbf{A}}, G_{m}\right\rangle$ is a $\mathcal{C}$-monotonic DS-space. 
Proof. Let $U \in D(X(\mathbf{A}))$. By definition, $U=\beta(a)$ for some $a \in A$. By Lemma 24 we have that $m_{R_{m}}(\beta(a))=\mathbf{m}_{G_{m}}(\beta(a))=\beta(m a) \in D(X(\mathbf{A}))$, i.e., $m_{R_{m}}(U), \mathbf{m}_{G_{m}}(U) \in$ $D(X(\mathbf{A}))$ for all $U \in D(X(\mathbf{A}))$.

Now we will show that for all $P \in X(\mathbf{A})$

$$
R_{m}(P)=\bigcap\left\{L_{\beta(a)}: m a \in P\right\} .
$$

Let $P \in X(\mathbf{A})$. It is clear that $R_{m}(P) \subseteq \bigcap\left\{L_{\beta(a)}: m a \in P\right\}$. On the other hand, let $Z \in \bigcap\left\{L_{\beta(a)}: m a \in P\right\}$, we will prove that $Z \in R_{m}(P)$. Suppose, contrary to our claim, that $Z \notin R_{m}(P)$. Then, there exists $a \in m^{-1}(P)$ such that $Z \cap \beta(a)=\emptyset$. By assumption, $Z \in L_{\beta(a)}$, i.e., $Z \cap \beta(a) \neq \emptyset$, which is a contradiction. Therefore, $Z \in R_{m}(P)$.

The indentity $G_{m}(P)=\bigcap\left\{\left(D_{\beta(a)}\right)^{c}: m a \notin P\right\}$ is proved similarly.

Lemma 31. Let $\langle\mathbf{A}, m\rangle \in \mathcal{M D S}$. Then $R_{m}(P)=\psi_{X(\mathbf{A})}\left(G_{m}(P)\right)$ and $G_{m}(P)=$ $\phi_{X(\mathbf{A})}\left(R_{m}(P)\right)$. Therefore the sets $R_{m}(P)$ and $G_{m}(P)$ are closed sets of the Galois connection $\left(\phi_{X(\mathbf{A})}, \psi_{X(\mathbf{A})}\right)$.

Proof. First, we will prove that $R_{m}(P)=\psi_{X(\mathbf{A})}\left(G_{m}(P)\right)$. Let $Z \in R_{m}(P)$. Then, $m^{-1}(P) \cap I_{\mathbf{A}}(Z)=\emptyset$. Let $Y \in G_{m}(P)$. By definition, $F_{Y} \subseteq m^{-1}(P)$ and we get that $F_{Y} \cap I_{\mathbf{A}}(Z)=\emptyset$. From Proposition $15, Y \cap Z \neq \emptyset$. Now, let $Z \in S(X(\mathbf{A}))$ and suppose that for all $Y \in G_{m}(P), Z \cap Y \neq \emptyset$. Let $a \in m^{-1}(P) \cap I_{\mathbf{A}}(Z)$. So, $[a) \subseteq m^{-1}(P)$ and by hypothesis $\widehat{[a)} \cap Z=\beta(a) \cap Z \neq \emptyset$. Then $a \notin I_{\mathbf{A}}(Z)$ which is a contradiction.

The other equality is proved analogously.

From now on, we consider a monotonic $D S$-space as an $\mathcal{S}$-monotonic $D S$-space. It is clear how we can construct one kind of space from the other, and there is no particular reason we have chosen $\mathcal{S}$-monotonic $D S$-spaces as our default other than to keep things simple and avoid repetition obtaining similar theorems and propositions.

Definition 32. Given $\langle\mathbf{A}, m\rangle \in \mathcal{M D S}$, the structure $\left\langle X(\mathbf{A}), \mathcal{T}_{\mathbf{A}}, R_{m}\right\rangle$ is the monotonic $D S$-space associated to $\langle\mathbf{A}, m\rangle$.

Definition 33. The algebra $\left\langle D(X), m_{R}\right\rangle$ is the monotonic distributive semilattice associated to the monotonic $D S$-space $\langle X, \mathcal{T}, R\rangle$.

Now, we are able to enunciate the representation theorem.

Theorem 34 (of Representation). Let $\langle\mathbf{A}, m\rangle \in \mathcal{M D S}$. Then, the structure $\left\langle\mathrm{Up}(X(\mathbf{A})), \cap, m_{R_{m}}, X(\mathbf{A})\right\rangle$ is a monotonic distributive semilattice and the map $\beta: A \rightarrow$ $\mathrm{Up}(X(\mathbf{A}))$ defined by

$$
\beta(a)=\{P \in X(\mathbf{A}): a \in P\}
$$

is an injective homomorphism of monotonic distributive semilattices.

Proof. It follows from Theorem 3 and the fact that for all $a \in A, m_{R_{m}}(\beta(a))=\beta(m a)$.

Corollary 35. Let $\langle\mathbf{A}, m\rangle \in \mathcal{M D S}$. Then, the map $\beta: A \rightarrow D(X(\mathbf{A}))$ defined by

$$
\beta(a)=\{P \in X(\mathbf{A}): a \in P\}
$$


is an isomorphism of monotonic distributive semilattices.

We note that if $\langle X, \mathcal{T}, R\rangle$ is a monotonic $D S$-space, then we have that $\left\langle X(D(X)), \mathcal{T}_{D(X)}, R_{m_{R}}\right\rangle$ is the monotonic space associated to $\left\langle D(X), m_{R}\right\rangle$. In [4] Celani has proved that the map

$$
H_{X}: X \rightarrow X(D(X))
$$

defined by

$$
H_{X}(x)=\{U \in D(X): x \in U\}
$$

is an homeomorphism between $D S$-spaces and an order isomorphism with respect to $\leq$. Now we introduce the following definition.

Definition 36. Let $\left\langle X_{1}, \mathcal{T}_{1}, R_{1}\right\rangle$ and $\left\langle X_{2}, \mathcal{T}_{2}, R_{2}\right\rangle$ be two monotonic $D S$-spaces. A map $f: X_{1} \rightarrow X_{2}$ is an isomorphism of DS-spaces if it satisfies,

(1) $f$ is a homeomorphism,

(2) $(x, Z) \in R_{1}$ if and only if $(f(x), f[Z]) \in R_{2}$, for all $x \in X_{1}$ and for each $Z \in$ $\mathcal{S}\left(X_{1}\right)$

where $f[Z]=\{f(z): z \in Z\}$.

Proposition 37. Let $\left\langle X_{1}, \mathcal{T}_{1}\right\rangle$ and $\left\langle X_{2}, \mathcal{T}_{2}\right\rangle$ be two DS-spaces and let $f: X_{1} \rightarrow X_{2}$ be a homeomorphism. Then, $f[Z] \in \mathcal{S}\left(X_{2}\right)$ for all $Z \in \mathcal{S}\left(X_{1}\right)$ and for all $S \in \mathcal{S}\left(X_{2}\right)$ there exists $Z \in \mathcal{S}\left(X_{1}\right)$ such that $S=f[Z]$.

Remark 38. Let $\langle X, \mathcal{T}\rangle$ be a $D S$-space. Then, $H_{X}[Z] \in \mathcal{S}(X(D(X))$ for all $Z \in S(X)$ and for all $S \in \mathcal{S}\left(X(D(X))\right.$ there exists $Z \in \mathcal{S}(X)$ such that $S=H_{X}[Z]$. Also, we have that $H_{X}[U]=\left\{H_{X}(u): u \in U\right\}=\beta_{D(X)}(U)$ for all $U \in D(X)$. Then,

$$
Z \cap U=\emptyset \Leftrightarrow H_{X}[Z] \cap \beta_{D(X)}(U)=\emptyset
$$

for all $Z \in \mathcal{S}(X)$ and $U \in D(X)$.

Theorem 39. Let $\langle X, \mathcal{T}, R\rangle$ be a monotonic DS-space. Then, the map $H_{X}: X \rightarrow$ $X(D(X))$ defined by

$$
H_{X}(x)=\{U \in D(X): x \in U\}
$$

is an isomorphism of monotonic DS-spaces.

Proof. By [4] and [1, it is only left to prove that $(x, Z) \in R$ iff $\left(H_{X}(x), H_{X}[Z]\right) \in R_{m_{R}}$.

$\Rightarrow)$ Let $Z \in \mathcal{S}(X)$ such that $(x, Z) \in R$. We will see that $H_{X}[Z] \cap \beta_{D(X)}(U) \neq \emptyset$ for all $U \in m_{R}^{-1}\left(H_{X}(x)\right)$. Let $U \in D(X)$ such that $U \in m_{R}^{-1}\left(H_{X}(x)\right)$, i.e., $m_{R}(U) \in H_{X}(x)$. Then, $x \in m_{R}(U)$. Since $Z \in R(x)$, we get that $Z \cap U \neq \emptyset$ and by Remark 38 , $H_{X}[Z] \cap \beta_{D(X)}(U) \neq \emptyset$. Therefore, $U \notin I_{D(X)}\left(H_{X}[Z]\right)$.

$\Leftarrow)$ Suppose that $\left(H_{X}(x), H_{X}[Z]\right) \in R_{m_{R}}$. Then, $H_{X}[Z] \cap \beta_{D(X)}(U) \neq \emptyset$ for all $U \in m_{R}^{-1}\left(H_{X}(x)\right)$, i.e., for all $U \in D(X)$ such that $x \in m_{R}(U)$. We will prove that $(x, Z) \in R$. To do so, suppose that $Z \notin R(x)$. From condition (4) of Definition 26 we have that there exists $U \in D(X)$ such that $x \in m_{R}(U)$ and $Z \cap U=\emptyset$. Then, by Remark 38. $H_{X}[Z] \cap \beta_{D(X)}(U)=\emptyset$, which is a contradiction. Therefore, $Z \in R(x)$. 
By the following result we get that the dual spaces of monotonic distributive semilattices are exactly those triples $\langle X, \mathcal{T}, R\rangle$, where $\langle X, \mathcal{T}\rangle$ is a $D S$-space, $R \subseteq X \times \mathcal{S}(X)$, $m_{R}(U) \in D(X)$, for all $U \in D(X)$, and $\langle X, \mathcal{T}, R\rangle$ satisfies any of the equivalent conditions of Theorem 41 .

Lemma 40. Let $\langle X, \mathcal{T}, R\rangle$ be a monotonic DS-space. Then $R(x)$ is an upset of $\langle\mathcal{S}(X), \subseteq\rangle$, i.e., for all $S, Z \in \mathcal{S}(X)$, and for all $x \in X$, if $S \subseteq Z$ and $S \in R(x)$, then $Z \in R(x)$.

Proof. Let $S, Z \in \mathcal{S}(X)$, and $x \in X$, such that $S \subseteq Z$ and $S \in R(x)$. If $Z \notin R(x)$, then by condition (4) of Definition 26, there exists $U \in D(X)$ such that $Z \cap U=\emptyset$ and $x \in m_{R}(U)$. But this implies that $S \cap U=\emptyset$ and $x \in m_{R}(U)$, which is impossible because $S \in R(x)$. Thus, $R(x)$ is an upset of $\langle\mathcal{S}(X), \subseteq\rangle$.

Theorem 41. Let $\langle X, \mathcal{T}\rangle$ be a DS-space. Consider a relation $R \subseteq X \times \mathcal{S}(X)$ such that $m_{R}(U)=\{x \in X: \forall Z \in R(x)[Z \cap U \neq \emptyset]\} \in D(X)$ for all $U \in D(X)$. Then, the following conditions are equivalent,

(1) $R(x)=\bigcap\left\{L_{U}: x \in m_{R}(U)\right.$ and $\left.U \in D(X)\right\}$ for all $x \in X$,

(2) For all $x \in X$ and for all $Z \in \mathcal{S}(X)$, if $\left(H_{X}(x), H_{X}[Z]\right) \in R_{m_{R}}$ then $(x, Z) \in R$,

(3) $m_{R}\left(Z^{c}\right)=\bigcup\left\{m_{R}(U): Z \subseteq U^{c}\right.$ and $\left.U \in D(X)\right\}$ for all $Z \in \mathcal{S}(X)$, and $R(x)$ is an upset of $\langle\mathcal{S}(X), \subseteq\rangle$ for all $x \in X$.

Proof. 1. $\Rightarrow 2$. It was proved in the previous theorem.

2. $\Rightarrow 1$. Let $x \in X$. The inclusion $R(x) \subseteq \bigcap\left\{L_{U}: x \in m_{R}(U)\right\}$ is clear. Let $Z \in \mathcal{S}(X)$ such that $Z \in \bigcap\left\{L_{U}: x \in m_{R}(U)\right\}$. We will prove that $\left(H_{X}(x), H_{X}[Z]\right) \in R_{m_{R}}$. Let $U \in D(X)$ such that $x \in m_{R}(U)$. Then, $Z \in L_{U}$, i.e., $Z \cap U \neq \emptyset$. By Remark 38 , we have that $H_{X}[Z] \cap \beta_{D(X)}(U) \neq \emptyset$. Thus, we have that for all $U \in D(X)$ such that $U \in m_{R}^{-1}\left(H_{X}(x)\right), H_{X}[Z] \cap \beta_{D(X)}(U) \neq \emptyset$, i.e., $U \notin I_{D(X)}\left(H_{X}[Z]\right)$. Therefore, $\left(H_{X}(x), H_{X}[Z]\right) \in R_{m_{R}}$ and by assumption, $Z \in R(x)$ and it follows that $\bigcap\left\{L_{U}: x \in\right.$ $\left.m_{R}(U)\right\} \subseteq R(x)$.

1. $\Rightarrow 3$. Let $x \in m_{R}\left(Z^{c}\right)$. Then, for all $S \in R(x)$ we have that $S \cap Z^{c} \neq \emptyset$. So, $Z \notin R(x)$. By assumption, $Z \notin \bigcap\left\{L_{U}: x \in m_{R}(U)\right\}$, i.e., there exists $U \in D(X)$ such that $x \in m_{R}(U)$ and $Z \cap U=\emptyset$. Thus, $x \in \bigcup\left\{m_{R}(U): Z \subseteq U^{c}\right.$ and $\left.U \in D(X)\right\}$. The other inclusion is trivial. The last part is a consequence of Lemma 40 .

3. $\Rightarrow$ 1. Let $x \in X$ and $Z \in \bigcap\left\{L_{U}: x \in m_{R}(U)\right.$ and $\left.U \in D(X)\right\}$. Suppose that $Z \notin$ $R(x)$. We will see that $x \in m_{R}\left(Z^{c}\right)$. On the contrary, suppose that $x \notin m_{R}\left(Z^{c}\right)$. Then, there exists $S \in R(x)$ such that $S \cap Z^{c}=\emptyset$. So, $S \subseteq Z$ and by assumption $Z \in R(x)$ which is a contradiction. Thus, $x \in m_{R}\left(Z^{c}\right)=\bigcup\left\{m_{R}(U): Z \subseteq U^{c}\right.$ and $\left.U \in D(X)\right\}$, i.e., there exists $U \in D(X)$ such that $x \in m_{R}(U)$ and $Z \cap U=\emptyset$, a contradiction. Therefore $Z \in R(x)$. The other inclusion is trivial.

4.1. Representation of homomorphisms. In [4] and [1] it was shown that there exists a duality between homomorphisms of distributive semilattices and certain binary relations called meet-relations. It is also known that $D S$-spaces with meet-relations form 
a category. Now, we shall study the representation of homomorphisms of monotonic distributive semilattices.

Let $S \subseteq X_{1} \times X_{2}$ be a binary relation. Consider the mapping $h_{S}: \mathcal{P}\left(X_{2}\right) \rightarrow \mathcal{P}\left(X_{1}\right)$ defined by

$$
h_{S}(U)=\left\{x \in X_{1}: S(x) \subseteq U\right\} .
$$

A meet-relation between two $D S$-spaces $\left\langle X_{1}, \mathcal{T}_{1}\right\rangle$ and $\left\langle X_{2}, \mathcal{T}_{2}\right\rangle$ was defined as a subset $S \subseteq X_{1} \times X_{2}$ satisfying the following conditions:

(1) For every $U \in D\left(X_{2}\right), h_{S}(U) \in D\left(X_{1}\right)$, and

(2) $S(x)=\bigcap\left\{U \in D\left(X_{2}\right): S(x) \subseteq U\right\}$ for all $x \in X_{1}$.

If $S$ is a meet-relation, then $h_{S}$ is a homomorphism between distributive semilattices.

On the other hand, let $\mathbf{A}, \mathbf{B} \in \mathcal{D S}$. Let $h: A \rightarrow B$ be a homomorphism. The binary relation $S_{h} \subseteq X(\mathbf{B}) \times X(\mathbf{A})$ defined by

$$
(P, Q) \in S_{h} \text { iff } h^{-1}[P] \subseteq Q
$$

is a meet-relation, where $h^{-1}[P]=\{a \in A: h(a) \in P\}$.

Definition 42. Let $\left\langle X_{1}, \mathcal{T}_{1}, R_{1}\right\rangle$ and $\left\langle X_{2}, \mathcal{T}_{2}, R_{2}\right\rangle$ be two monotonic $D S$-spaces. Let us consider a meet-relation $S \subseteq X_{1} \times X_{2}$. We say that $S$ is a monotonic meet-relation if for all $x \in X_{1}$ and every $U \in D\left(X_{2}\right)$ it satisfies

$$
U^{c} \in R_{2}[S(x)] \text { iff } S^{-1}\left[U^{c}\right] \in R_{1}(x)
$$

where $R_{2}[S(x)]=\left\{Z \in \mathcal{S}\left(X_{2}\right): \exists y \in S(x)\left[(y, Z) \in R_{2}\right]\right\}$.

Remark 43. Note that if $S \subseteq X_{1} \times X_{2}$ is a meet-relation between two $D S$-spaces $\left\langle X_{1}, \mathcal{T}_{1}\right\rangle$ and $\left\langle X_{2}, \mathcal{T}_{2}\right\rangle$, then $S^{-1}\left[U^{c}\right]=h_{S}(U)^{c} \in \mathcal{S}\left(X_{1}\right)$.

Proposition 44. The condition (4.5) is equivalent to the condition

$$
h_{S}\left(m_{R_{2}}(U)\right)=m_{R_{1}}\left(h_{S}(U)\right)
$$

for all $U \in D_{\mathcal{K}_{2}}\left(X_{2}\right)$, i.e., the mapping $h_{S}: D\left(X_{2}\right) \rightarrow D\left(X_{1}\right)$ is a homomorphism of monotonic distributive semilattices.

Proof. $\Rightarrow$ ) Suppose that for all $x \in X_{1}$ and every $U \in D\left(X_{2}\right), U^{c} \in R_{2}[S(x)]$ if and only if $S^{-1}\left[U^{c}\right] \in R_{1}(x)$. Let $x \in h_{S}\left(m_{R_{2}}(U)\right)$, i.e., $S(x) \subseteq m_{R_{2}}(U)$. Then, for all $y \in S(x)$ we have that $y \in m_{R_{2}}(U)$. So, for all $y \in S(x)$ and for all $Z \in R_{2}(y)$ we have that $Z \cap U \neq \emptyset$. Then, for all $y \in S(x), U^{c} \notin R_{2}(y)$. Thus, $U^{c} \notin R_{2}[S(x)]$. By hypothesis, $S^{-1}\left[U^{c}\right] \notin R_{1}(x)$. Therefore, $x \in m_{R_{1}}\left(S^{-1}\left[U^{c}\right]^{c}\right)=m_{R_{1}}\left(h_{S}(U)\right)$. The other inclusion is obtained reverting the implications.

$\Leftarrow)$ Suppose that $h_{S}$ is a homomorphism. Let $U^{c} \in R_{2}[S(x)]$. Then, there exists $y \in S(x)$ such that $U^{c} \in R_{2}(y)$. So, $y \notin m_{R_{2}}(U)$. Thus, $S(x) \nsubseteq m_{R_{2}}(U)$, i.e., $x \notin$ $h_{S}\left(m_{R_{2}}(U)\right)$. By hypothesis, $x \notin m_{R_{1}}\left(h_{S}(U)\right)$, i.e., there exists $Z \in R_{1}(x)$ such that $Z \cap h_{S}(U)=\emptyset$. We have that $Z \subseteq h_{S}(U)^{c}$ and since $h_{S}(U)^{c} \in \mathcal{S}(X)$ we have that $S^{-1}\left[U^{c}\right]=h_{S}(U)^{c} \in R_{1}(x)$. The other implication is obtained similarly. 
Now, we will study the composition of monotonic meet-relations. Let $X_{1}, X_{2}$ and $X_{3}$ be sets. Let us consider two relations $S_{1} \subseteq X_{1} \times X_{2}$ and $S_{2} \subseteq X_{2} \times X_{3}$. Then, the composition of $S_{1}$ and $S_{2}$ is the relation $S_{2} \circ S_{1} \subseteq X_{1} \times X_{3}$ defined by

$$
S_{2} \circ S_{1}=\left\{(x, z) \in X_{1} \times X_{3}: \exists y \in X_{2}\left[(x, y) \in S_{1} \text { and }(y, z) \in S_{2}\right\} .\right.
$$

Proposition 45. Let $\left\langle X_{1}, \mathcal{T}_{1}, R_{1}\right\rangle,\left\langle X_{2}, \mathcal{T}_{2}, R_{2}\right\rangle$ and $\left\langle X_{3}, \mathcal{T}_{3}, R_{3}\right\rangle$ be three monotonic DSspaces. Let us consider two monotonic meet-relations $S_{1} \subseteq X_{1} \times X_{2}$ and $S_{2} \subseteq X_{2} \times X_{3}$. Then, $S_{3}=S_{2} \circ S_{1} \subseteq X_{1} \times X_{3}$ is a monotonic meet-relation.

Proof. It follows from the fact that $h_{S_{3}}(U)=h_{S_{2} \circ S_{1}}(U)=h_{S_{1}} \circ h_{S_{2}}(U)$ for all $U \in$ $D_{\mathcal{K}_{3}}\left(X_{3}\right)$, definition 42 and proposition 44 .

Proposition 46. Let $\langle X, \mathcal{T}, R\rangle$ be a monotonic DS-space. The specialization dual order $\leq \subseteq X \times X$ is a monotonic meet-relation.

Proof. $\Rightarrow)$ Let $U \in D(X)$ and suppose that $U^{c} \in R([x))$. Then, there exists $y \geq x$ such that $U^{c} \in R(y)$ and since $R(y) \subseteq R(x)$, we have that $U^{c} \in R(x)$. As $U^{c}$ is a downset, $\leq^{-1}\left[U^{c}\right]=U^{c}$.

The other implication is trivial.

So, monotonic $D S$-spaces with monotonic meet-relations form a category where the identity arrow is the specialization dual order. We will denote this category by $\mathcal{M D S R}$.

Proposition 47. Let $\left\langle\mathbf{A}, m_{\mathbf{A}}\right\rangle,\left\langle\mathbf{B}, m_{\mathbf{B}}\right\rangle \in \mathcal{M D S}$.

(1) Let $h: A \rightarrow B$ be a monotonic homomorphism. Then, the meet-relation $S_{h}$ satisfies condition (4.5).

(2) Let $h: A \rightarrow B$ be a homomorphism and suppose that the meet-relation $S_{h}$ satisfies condition (4.5). Then, $h$ is monotonic.

Proof. 1. Suppose that $h$ is a monotonic homomorphism. So, it is easy to see that $h_{S_{h}}\left(\beta_{\mathbf{A}}(a)\right)=\beta_{\mathbf{B}}(h(a))$ for all $a \in A$. Then, we have

$$
\begin{aligned}
h_{S_{h}}\left(m_{R_{m_{\mathbf{A}}}} \beta_{\mathbf{A}}(a)\right) & =h_{S_{h}}\left(\beta_{\mathbf{A}}\left(m_{\mathbf{A}} a\right)\right)=\beta_{\mathbf{B}}\left(h\left(m_{\mathbf{A}} a\right)\right) \\
& =\beta_{\mathbf{B}}\left(m_{\mathbf{B}} h(a)\right)=m_{R_{m_{\mathbf{B}}}}\left(\beta_{\mathbf{B}}(h(a))\right) \\
& =m_{R_{m_{\mathbf{B}}}}\left(h_{S_{h}}\left(\beta_{\mathbf{A}}(a)\right)\right)
\end{aligned}
$$

for all $a \in A$.

2. Suppose that $h$ is a homomorphism and that $S_{h}$ satisfies condition (4.5). Then, $h_{S_{h}}\left(\beta_{\mathbf{A}}(a)\right)=\beta_{\mathbf{B}}(h(a))$ for all $a \in A$. So, we have

$$
\begin{aligned}
\beta_{\mathbf{B}}\left(h\left(m_{\mathbf{A}} a\right)\right) & =h_{S_{h}}\left(\beta_{\mathbf{A}}\left(m_{\mathbf{A}} a\right)\right)=h_{S_{h}}\left(m_{R_{m_{\mathbf{A}}}} \beta_{\mathbf{A}}(a)\right) \\
& =m_{R_{m_{\mathbf{B}}}}\left(h_{S_{h}}\left(\beta_{\mathbf{A}}(a)\right)\right)=m_{R_{m_{\mathbf{B}}}}\left(\beta_{\mathbf{B}}(h(a))\right) \\
& =\beta_{\mathbf{B}}\left(m_{\mathbf{B}} h(a)\right)
\end{aligned}
$$

and since $\beta_{\mathbf{B}}$ is an injective function, we get that $h\left(m_{\mathbf{A}} a\right)=m_{\mathbf{B}} h(a)$ for all $a \in A$.

From Theorem 39 and Proposition 44, we conclude that the functor $\mathbb{D}: \mathcal{M D S R} \rightarrow$ $\mathcal{M D S H}$ defined by 
(1) $\mathbb{D}(X)=\left\langle D(X), m_{R}\right\rangle$ if $\langle X, \mathcal{T}, R\rangle$ is a $D S$-space,

(2) $\mathbb{D}(S)=h_{S}$ if $S$ is a monotonic meet-relation

is a contravariant functor. By Theorem 34, Corollary 35 and Proposition 47, we conclude that the functor $\mathbb{X}: \mathcal{M D S H} \rightarrow \mathcal{M D S R}$ defined by

(1) $\mathbb{X}(\mathbf{A})=\left\langle X(\mathbf{A}) ; \mathcal{T}_{A}, R_{m}\right\rangle$ if $\langle\mathbf{A}, m\rangle$ is a monotonic distributive semilattice,

(2) $\mathbb{X}(h)=S_{h}$ if $h$ is homomorphism of monotonic distributive semilattices is a contravariant functor. Therefore, we give the following result.

Corollary 48. The categories $\mathcal{M D S H}$ and $\mathcal{M D S R}$ are dually equivalent.

\section{Applications of the Duality}

In this section we consider some applications of the duality. We will consider some important subclasses and show how our new duality extends the one developed in [3] for Boolean algebras.

5.1. Additional conditions. Now we will see how some additional conditions affect the relations associated to the monotonic operator.

The following formulas are $\pi$ - and $\sigma$-canonical, i.e., their validity is preserved under taking $\pi$ - and $\sigma$-canonical extensions.

Proposition 49. Let $\langle A, m\rangle \in \mathcal{M D S}$. Then,

(1) $m 1=1$ iff $\forall P \in X(\mathbf{A})\left[\emptyset \notin R_{m}(P)\right]$ iff $\forall P \in X(\mathbf{A})\left[X(\mathbf{A}) \in G_{m}(P)\right]$;

(2) $m 0=0$ iff $\forall P \in X(\mathbf{A})\left[X(\mathbf{A}) \in R_{m}(P)\right]$ iff $\forall P \in X(\mathbf{A})\left[\emptyset \notin G_{m}(P)\right]$;

(3) $\forall a \in A[m a \leq a]$ iff $\forall P \in X(\mathbf{A})\left[\alpha\left(P^{c}\right)=(P] \in R_{m}(P)\right]$ iff $\forall P \in X(\mathbf{A}) \forall Y \in G_{m}(P)[P \in Y]$

(4) $\forall a \in A[a \leq m a]$ iff $\forall P \in X(\mathbf{A}) \forall Z \in R_{m}(P)[P \in Z]$ iff $\forall P \in X(\mathbf{A})\left[\hat{P}=[P) \in G_{m}(P)\right]$.

Proof. 1. Suppose that $m 1=1$ and suppose that there exists $P \in X(\mathbf{A})$ such that $\emptyset \in R_{m}(P)$. Then, $m 1=1 \in P$ and $m^{-1}(P) \cap I_{\mathbf{A}}(\emptyset)=m^{-1}(P) \cap A=\emptyset$ and it follows that $m^{-1}(P)=\emptyset$ which is a contradiction.

Now, suppose that for all $P \in X(\mathbf{A})$ we have $\emptyset \notin R_{m}(P)$ and suppose that there exists $P \in X(\mathbf{A})$ such that $X(\mathbf{A}) \notin G_{m}(P)$. Then, $F_{X(\mathbf{A})}=\{1\} \nsubseteq m^{-1}(P)$, i.e., $1 \notin m^{-1}(P)$. Since $P$ is an upset, $m^{-1}(P)=\emptyset$. So, we have that $m^{-1}(P) \cap A=m^{-1}(P) \cap I_{\mathbf{A}}(\emptyset)=\emptyset$ and by definition $\emptyset \in R_{m}(P)$ which is a contradiction.

Suppose that for all $P \in X(\mathbf{A})$ we have $X(\mathbf{A}) \in G_{m}(P)$ and suppose that $m 1 \neq 1$. Then, there exists $P \in X(\mathbf{A})$ such that $m 1 \notin P$. So, we have that $F_{X(\mathbf{A})}=\{1\} \nsubseteq$ $m^{-1}(P)$ which is a contradiction.

2. The proof is similar to 1 .

3. Suppose that $m a \leq a$ for all $a \in A$ and that there exists $P \in X(\mathbf{A})$ such that $(P] \notin R_{m}(P)$. Then, $m^{-1}(P) \cap P^{c} \neq \emptyset$. So, there exists $a \in A$ such that $m a \in P$ and $a \notin P$, which is a contradiction.

Now, suppose that for all $P \in X(\mathbf{A})$ we have $(P] \in R_{m}(P)$ and suppose that there exists $P \in X(\mathbf{A})$ and there exists $Y \in G_{m}(P)$ such that $P \notin Y$. Then, $F_{Y} \subseteq m^{-1}(P)$ 
and there exists $a \in F_{Y}$ such that $a \notin P$. So, $a \in m^{-1}(P) \cap P^{c}$ and it follows that $(P] \notin R_{m}(P)$ which is a contradiction.

Suppose that for all $P \in X(\mathbf{A})$ and for all $Y \in G_{m}(P)$ we have $P \in Y$ and suppose that $m a \not \leq a$. Then, there exists $P \in X(\mathbf{A})$ such that $m a \in P$ and $a \notin P$. So, we have that $[a) \subseteq m^{-1}(P)$ but $P \notin \widehat{[a]}$ which is a contradiction.

4. The proof is similar to 3 .

Now, we will characterize the dual spaces of monotonic distributive meet-semilattices satisfying condition $\left(\mathbf{4}_{\square}\right) m a \leq m^{2} a$ or condition $\left(\mathbf{4}_{\diamond}\right) m^{2} a \leq m a$ for every element $a$. We will see that condition $\mathbf{4}_{\square}$ is $\sigma$-canonical and that condition $\mathbf{4}_{\diamond}$ is $\pi$-canonical.

Let $\langle X, \mathcal{T}, R\rangle$ be a monotonic $D S$-space. For any $U \in \operatorname{Up}(X)$, we define the operator $m_{R}^{2}: \mathrm{Up}(X) \rightarrow \mathrm{Up}(X)$ by $m_{R}^{2}(U)=m_{R}\left(m_{R}(U)\right)$.

Remark 50. Let $\langle X, \mathcal{T}, R\rangle$ be a monotonic $D S$-space and let $Z \in \mathcal{S}(X)$. Then recall that $m_{R}\left(Z^{c}\right)^{c}=\bigcap\left\{m_{R}(U)^{c}: U \in D(X)\right.$ and $\left.Z \subseteq U^{c}\right\}$.

Proposition 51. Let $\langle\mathbf{A}, m\rangle \in \mathcal{M D S}$ such that $m^{2} a \leq m a$ for all $a \in A$. Then, $m_{R_{m}}^{2}(U) \subseteq m_{R_{m}}(U)$ for all $U \in \mathrm{Up}(X(\mathbf{A}))$, i.e., $\mathbf{4}_{\diamond}$ is $\pi$-canonical.

Proof. Let $A \in \mathcal{M D S}$ such that $m^{2} a \leq m a$ for all $a \in A$. Then, for all $U \in D(X(\mathbf{A}))$ we have that $m_{R_{m}}^{2}(U) \subseteq m_{R_{m}}(U)$. First, we will see that $m_{R_{m}}^{2}\left(Z^{c}\right) \subseteq m_{R_{m}}\left(Z^{c}\right)$ for all $Z \in$ $\mathcal{S}(X(\mathbf{A}))$. Let $P \in m_{R_{m}}^{2}\left(Z^{c}\right)$. So, we get that $m_{R_{m}}\left(Z^{c}\right)^{c} \notin R_{m}(P)$. Suppose that $P \notin$ $m_{R_{m}}\left(Z^{c}\right)$. Then, we get that $Z \in R_{m}(P)$. Since $R_{m}(P)=\bigcap\left\{L_{U}: U \in D(X)\right.$ and $P \in$ $\left.m_{R_{m}}(U)\right\}$, there exists $U \in D(X)$ such that $P \in m_{R_{m}}(U)$ and $m_{R_{m}}\left(Z^{c}\right)^{c} \cap U=\emptyset$. By the previous remark, there exists $V \in D(X)$ such that $Z \subseteq V^{c}$ and $m_{R_{m}}(V)^{c} \cap U=\emptyset$. Thus, $U \subseteq m_{R_{m}}(V)$ and by hypothesis $P \in m_{R_{m}}(U) \subseteq m_{R_{m}}^{2}(V) \subseteq m_{R_{m}}(V)$. Since $P \in m_{R_{m}}(V)$ and $Z \in R_{m}(P)$ we get that $Z \cap V \neq \emptyset$ which is a contradiction.

Now, we will see that $m_{R_{m}}^{2}(U) \subseteq m_{R_{m}}(U)$ for all $U \in \mathrm{Up}(X(\mathbf{A}))$. Let $P \in m_{R_{m}}^{2}(U)$ and suppose that $P \notin m_{R_{m}}(U)$. Then, there exists $Z \in R_{m}(P)$ such that $Z \cap U=\emptyset$. So, $U \subseteq Z^{c}$ and we get that $m_{R_{m}}(U) \subseteq m_{R_{m}}\left(Z^{c}\right)$. Thus, $m_{R_{m}}(U) \cap m_{R_{m}}\left(Z^{c}\right)^{c}=\emptyset$ and since $P \in m_{R_{m}}^{2}(U)$ we get that $m_{R_{m}}\left(Z^{c}\right)^{c} \notin R_{m}(P)$. Therefore $P \in m_{R_{m}}^{2}\left(Z^{c}\right) \subseteq m_{R_{m}}\left(Z^{c}\right)$ which is a contradiction because $Z \in R_{m}(P)$.

Let $\langle X, \mathcal{T}, R\rangle$ be a monotonic $D S$-space. We will define a relation $\bar{R} \subseteq \mathcal{S}(X) \times \mathcal{S}(X)$ by

$$
(S, Z) \in \bar{R} \Leftrightarrow \forall x \in S(x, Z) \in R .
$$

We define $R^{2} \subseteq X \times \mathcal{S}(X)$ as follows

$$
(x, Z) \in R^{2} \Leftrightarrow \exists S \in \mathcal{S}(X) \text { such that }(x, S) \in R \text { and }(S, Z) \in \bar{R} .
$$

Definition 52. Let $\langle X, \mathcal{T}, R\rangle$ be a monotonic $D S$-space. The relation $R$ is transitive if and only if for all $x \in X$ and for all $Z \in \mathcal{S}(X)$ if $(x, Z) \in R^{2}$ then $(x, Z) \in R$.

Definition 53. Let $\langle X, \mathcal{T}, R\rangle$ be a monotonic $D S$-space. The relation $R$ is weakly dense if and only if for all $x \in X$ and for all $Z \in \mathcal{S}(X)$ if $(x, Z) \in R$ then $(x, Z) \in R^{2}$. 
Corollary 54. Let $\langle A, m\rangle \in \mathcal{M D S}$. Then, $I_{\mathbf{A}}\left(m_{R_{m}}\left(\alpha(I)^{c}\right)^{c}\right)=(m(I)]$ where $m(I)=$ $\{m a: a \in I\}$.

Lemma 55. Let $A \in \mathcal{M D S}$. Then for all $P \in X(\mathbf{A})$ and $I \in \operatorname{Id}(\mathbf{A}),(P, \alpha(I)) \in R_{m}^{2}$ if and only if $I \subseteq\left\{a \in A: m^{2} a \in P^{c}\right\}$.

Proof. $\Rightarrow)$ Suppose that $(P, \alpha(I)) \in R_{m}^{2}$ and let $a \in I$. Suppose that $m^{2} a \in P$. Then, there exists $J \in \operatorname{Id}(\mathbf{A})$ such that $(P, \alpha(J)) \in R_{m}$ and $(\alpha(J), \alpha(I)) \in \bar{R}_{m}$. So, $m^{-1}(P) \cap$ $J=\emptyset$ and $m a \notin J$. Thus, there exists $Q \in \alpha(J)$ such that $m a \in Q$. Therefore, $(Q, \alpha(I)) \in R_{m}$ and $a \notin I$ which is a contradiction.

$\Leftarrow)$ Suppose that $I \subseteq\left\{a \in A: m^{2} a \in P^{c}\right\}$. We will prove that $m_{R_{m}}\left(\alpha(I)^{c}\right)^{c} \in$ $R_{m}(P)$. Since $I_{\mathbf{A}}\left(m_{R_{m}}\left(\alpha(I)^{c}\right)^{c}\right)=(m(I)]$, suppose that there exists $a \in A$ such that $a \in$ $m^{-1}(P) \cap(m(I)]$. So, $m a \in P$ and there exists $b \in I$ such that $a \leq m b$. Then, $m a \leq m^{2} b$ and we get that $m^{2} b \in P \cap P^{c}$ which is a contradiction. Therefore, $m_{R_{m}}\left(\alpha(I)^{c}\right)^{c} \in R_{m}(P)$ and $\left(m_{R_{m}}\left(\alpha(I)^{c}\right)^{c}, \alpha(I)\right) \in \bar{R}_{m}$, i.e., $(P, \alpha(I)) \in R_{m}^{2}$.

Proposition 56. Let $A \in \mathcal{M D S}$. Then $m a \leq m^{2} a$ for all $a \in A$ if and only if $R_{m}$ is transitive.

Proof. $\Rightarrow)$ Suppose that $m a \leq m^{2} a$ for all $a \in A$ and that $(P, Z) \in R_{m}^{2}$. Then, $I_{\mathbf{A}}(Z) \subseteq$ $\left\{a \in A: m^{2} a \in P^{c}\right\}$. Suppose that $m^{-1}(P) \cap I_{\mathbf{A}}(Z) \neq \emptyset$. We get that there exists $a \in A$ such that $m a \in P$ and $a \in I_{\mathbf{A}}(Z)$. Therefore $m^{2} a \notin P$ and $m^{2} a \in P$ which is a contradiction. Since $m^{-1}(P) \cap I_{\mathbf{A}}(Z)=\emptyset$ we get that $(P, Z) \in R_{m}$.

$\Leftarrow)$ Suppose that $R_{m}$ is transitive and suppose that there exists $a \in A$ such that $m a \not \leq m^{2} a$. Then, there exists $P \in X(\mathbf{A})$ such that $m a \in P$ and $m^{2} a \notin P$. So, $(a] \subseteq$ $\left\{a \in A: m^{2} a \in P^{c}\right\}$ and by Lemma 55 , $(P, \alpha(a)) \in R_{m}^{2}$. We get that $(P, \alpha(a)) \in R_{m}$, i.e., $m a \notin P$, which is a contradiction.

Proposition 57. Let $A \in \mathcal{M D S}$. Then $m^{2} a \leq m a$ for all $a \in A$ if and only if $R_{m}$ is weakly dense.

Proof. $\Rightarrow)$ Suppose that $m^{2} a \leq m a$ for all $a \in A$ and that $(P, Z) \in R_{m}$. We will prove that $I_{\mathbf{A}}(Z) \subseteq\left\{a \in A: m^{2} a \in P^{c}\right\}$. Let $a \in I_{\mathbf{A}}(Z)$. Since $m^{-1}(P) \cap I_{\mathbf{A}}(Z)=\emptyset$ we get that $m a \notin P$ and therefore $m^{2} a \notin P$. By Lemma 55 , $(P, Z) \in R_{m}^{2}$.

$\Leftarrow)$ Suppose that $R_{m}$ is weakly dense. Suppose that there exists $a \in A$ such that $m^{2} a \not \leq m a$. Then, there exists $P \in X(\mathbf{A})$ such that $m^{2} a \in P$ and $m a \notin P$. Then, $(P, \alpha(a)) \in R_{m}$. So, $(P, \alpha(a)) \in R_{m}^{2}$ and, by Lemma 55, (a] $\subseteq\left\{a \in A: m^{2} a \in P^{c}\right\}$, i.e., $m^{2} a \notin P$, which is a contradiction.

For the sake of completeness we add the corresponding definitions and theorems for $\mathcal{C}$-monotonic $D S$-spaces.

Let $\langle X, \mathcal{T}, G\rangle$ be a $\mathcal{C}$-monotonic $D S$-space. For any $U \in \mathrm{Up}(X)$ we define the operator $\mathbf{m}_{G}^{2}: \mathrm{Up}(X) \rightarrow \mathrm{Up}(X)$ by $\mathbf{m}_{G}^{2}(U)=\mathbf{m}_{G}\left(\mathbf{m}_{G}(U)\right)$.

Remark 58. Let $\langle X, \mathcal{T}, G\rangle$ be a $\mathcal{C}$-monotonic $D S$-space and $Y \in \mathcal{C}(X)$. Recall that $\mathbf{m}_{G}(Y)=\bigcap\left\{\mathbf{m}_{G}(U): U \in D(X)\right.$ and $\left.Y \subseteq U\right\}$. 
Proposition 59. Let $\langle\mathbf{A}, m\rangle \in \mathcal{M D S}$ such that $m a \leq m^{2} a$ for all $a \in A$. Then, $\mathbf{m}_{G_{m}}(U) \subseteq \mathbf{m}_{G_{m}}^{2}(U)$ for all $U \in \mathrm{Up}(X(\mathbf{A}))$, i.e., $\mathbf{4}_{\square}$ is $\sigma$-canonical.

Proof. Let $A \in \mathcal{M D S}$ such that $m a \leq m^{2} a$ for all $a \in A$. Then, for all $U \in D(X(\mathbf{A}))$ we have that $\mathbf{m}_{G_{m}}(U) \subseteq \mathbf{m}_{G_{m}}^{2}(U)$. First, we will see that $\mathbf{m}_{G_{m}}(Y) \subseteq \mathbf{m}_{G_{m}}^{2}(Y)$ for all $Y \in \mathcal{C}(X(\mathbf{A}))$. Let $P \in \mathbf{m}_{G_{m}}(Y)$. So, we get that $Y \in G_{m}(P)$. Suppose that $P \notin \mathbf{m}_{G_{m}}^{2}(Y)$. Then, we get that $\mathbf{m}_{G_{m}}(Y) \notin G_{m}(P)$. Since $G_{m}(P)=\bigcap\left\{\left(D_{U}\right)^{c}\right.$ : $U \in D(X)$ and $\left.P \notin \mathbf{m}_{G_{m}}(U)\right\}$, there exists $U \in D(X)$ such that $P \notin \mathbf{m}_{G_{m}}(U)$ and $\mathbf{m}_{G_{m}}(Y) \subseteq U$. By the previous remark, there exists $V \in D(X)$ such that $Y \subseteq V$ and $\mathbf{m}_{G_{m}}(Y) \subseteq \mathbf{m}_{G_{m}}(V) \subseteq U$. Thus, $P \in \mathbf{m}_{G_{m}}(V)$ and by hypothesis $P \in \mathbf{m}_{G_{m}}^{2}(V) \subseteq$ $\mathbf{m}_{G_{m}}(U)$ which is a contradiction.

Now, we will see that $\mathbf{m}_{G_{m}}(U) \subseteq \mathbf{m}_{G_{m}}^{2}(U)$ for all $U \in \operatorname{Up}(X(\mathbf{A}))$. Let $P \in \mathbf{m}_{G_{m}}(U)$. Then, there exists $Y \in G_{m}(P)$ such that $Y \subseteq U$. So, $\mathbf{m}_{G_{m}}(Y) \subseteq \mathbf{m}_{G_{m}}(U)$ and we get that $P \in \mathbf{m}_{G_{m}}(Y) \subseteq \mathbf{m}_{G_{m}}^{2}(Y) \subseteq \mathbf{m}_{G_{m}}^{2}(U)$. Thus, $P \in \mathbf{m}_{G_{m}}^{2}(U)$.

Let $\langle X, \mathcal{T}, G\rangle$ be a $\mathcal{C}$-monotonic $D S$-space. We will define a relation $\bar{G} \subseteq \mathcal{C}(X) \times \mathcal{C}(X)$ by

$$
(Y, C) \in \bar{G} \Leftrightarrow \forall x \in Y(x, C) \in G .
$$

We define $G^{2} \subseteq X \times \mathcal{C}(X)$ as follows

$$
(x, Y) \in G^{2} \Leftrightarrow \exists C \in \mathcal{C}(X) \text { such that }(x, C) \in G \text { and }(C, Y) \in \bar{G} .
$$

Definition 60. Let $\langle X, \mathcal{T}, G\rangle$ be a $\mathcal{C}$ - monotonic $D S$-space. The relation $G$ is transitive if and only if for all $x \in X$ and for all $Y \in \mathcal{C}(X)$ if $(x, Y) \in G^{2}$ then $(x, Y) \in G$.

Definition 61. Let $\langle X, \mathcal{T}, G\rangle$ be a a $\mathcal{C}$ - monotonic $D S$-space. The relation $G$ is weakly dense if and only if for all $x \in X$ and for all $Y \in \mathcal{C}(X)$ if $(x, Y) \in G$ then $(x, Y) \in G^{2}$.

Lemma 62. Let $A \in \mathcal{M D S}$ and $F \in \mathrm{Fi}(\mathbf{A})$. Then, $F_{\mathbf{m}_{G_{m}}(\hat{F})}=[m(F))$ where $m(F)=$ $\{m a: a \in F\}$.

Lemma 63. Let $A \in \mathcal{M D S}$. Then for all $P \in X(\mathbf{A})$ and $F \in \operatorname{Fi}(\mathbf{A}),(P, \hat{F}) \in G_{m}^{2} \Leftrightarrow$ $F \subseteq\left\{a \in A: m^{2} a \in P\right\}$.

Proposition 64. Let $A \in \mathcal{M D S}$. Then $m a \leq m^{2} a$ for all $a \in A$ if and only if $G_{m}$ is weakly dense.

Proposition 65. Let $A \in \mathcal{M D S}$. Then $m^{2} a \leq m a$ for all $a \in A$ if and only if $G_{m}$ is transitive.

5.2. Modal distributive semilattices. In this section we consider distributive semilattices endowed with a normal modal operator, i.e., a function that preserves the greatest element and finite meets.

Definition 66. A modal distributive semilattice is an algebra $\langle\mathbf{A}, m\rangle$ where $\mathbf{A}$ is a distributive semilattice and $m: A \rightarrow A$ is an operator that verifies the following conditions:

(1) $m 1=1$,

(2) $m(a \wedge b)=m a \wedge m b$ for all $a, b \in A$. 
It is clear that $m$ is a homomorphism and that a modal distributive semilattice is a monotonic distributive semilattice. Thus, a modal operator $m$ could be represented by means of an adequate multirelation defined in the dual space and by a meet-relation. Now we are going to identify what additional conditions must satisfy this multirelation.

Remark 67. Let $\langle X, \mathcal{T}, R\rangle$ be a monotonic $D S$-space. Since $(x]=\bigcap\{U \in \mathcal{K} \mathcal{O}(X): x \in$ $U\}$ and $\mathcal{K O}(X)$ is a basis, we have that $(x] \in \mathcal{S}(X)$ for each $x \in X$.

Remark 68. Given a modal distributive semilattice $\langle\mathbf{A}, m\rangle$, we note that $m^{-1}(F) \in$ $\operatorname{Fi}(\mathbf{A})$ for all $F \in \operatorname{Fi}(\mathbf{A})$. We also note that $I_{\mathbf{A}}((Q])=Q^{c}$ for all $Q \in X(\mathbf{A})$.

Definition 69. A monotonic $D S$-space $\langle X, \mathcal{T}, R\rangle$ is called normal if for any $x \in X$ and for every $Z \in \mathcal{S}(X)$ such that $Z \in R(x)$ there exists $z \in Z$ such that $(z] \in R(x)$.

Note that in every normal monotonic $D S$-space $\langle X, \mathcal{T}, R\rangle$ for all $x \in X, \emptyset \notin R(x)$.

Proposition 70. Let $\langle\mathbf{A}, m\rangle$ be a monotonic distributive semilattice. Then $\langle\mathbf{A}, m\rangle$ is a modal distributive semilattice iff $\left\langle X(\mathbf{A}), \mathcal{T}_{\mathbf{A}}, R_{m}\right\rangle$ is a normal monotonic DS-space.

Proof. $\Rightarrow)$ Let $(P, \alpha(I)) \in R_{m}$. Then, $m^{-1}(P) \cap I=\emptyset$. Since $\langle\mathbf{A}, m\rangle$ is a modal distributive semilattice, we have that $m^{-1}(P) \in \mathrm{Fi}(\mathbf{A})$. So, there exists $Q \in X(\mathbf{A})$ such that $m^{-1}(P) \subseteq Q$ and $Q \cap I=\emptyset$. Thus, $Q \in \alpha(I)$ and it is easy to see that $m^{-1}(P) \cap Q^{c}=\emptyset$ and therefore, $(P,(Q]) \in R_{m}$.

$\Leftarrow)$ Let $\left\langle X(\mathbf{A}), \mathcal{T}_{\mathbf{A}}, R_{m}\right\rangle$ be a normal monotonic $D S$-space. Suppose that there exist $a, b \in A$ such that $m a \wedge m b \not \leq m(a \wedge b)$. Then, there exists $P \in X(\mathbf{A})$ such that $m a \wedge m b \in P$ but $m(a \wedge b) \notin P$. Note that $m a \wedge m b \leq m a \in P$ and $m a \wedge m b \leq m b \in P$. So, we have that $(P, \alpha(a \wedge b)) \in R_{m}$ and since $\left\langle X(\mathbf{A}), \mathcal{T}_{\mathbf{A}}, R_{m}\right\rangle$ is a normal monotonic $D S$-space, there exists $Q \in \alpha(a \wedge b)$ such that $(P,(Q]) \in R_{m}$. From $I_{\mathbf{A}}((Q])=Q^{c}$ we get that $m^{-1}(P) \cap Q^{c}=\emptyset$. Thus, $a, b \in m^{-1}(P) \subseteq Q$ and, since $Q$ is a filter, we have that $a \wedge b \in Q$. Hence, $Q \cap(a \wedge b] \neq \emptyset$ which contradicts the fact that $Q \in \alpha(a \wedge b)$. Therefore $\langle\mathbf{A}, m\rangle$ is a modal distributive semilattice.

Since a normal modal operator is also a homomorphism of meet semilattices, we can also interpret it through a meet-relation in the dual space. We will show the relationship between the multirelation and the meet-relation associated to the same operator.

Let $\langle X, \mathcal{T}\rangle$ be a $D S$-space and let $S \subseteq X \times X$ be a meet-relation. Let $\mathrm{m}_{S}: \operatorname{Up}(X) \rightarrow$ $\mathrm{Up}(X)$ be the operator defined by

$$
\mathrm{m}_{S}(U)=\{x \in X: S(x) \subseteq U\}
$$

where $S(x)=\{y \in X:(x, y) \in S\}$. We define a multirelation $R_{S} \subseteq X \times \mathcal{S}(X)$ by

$$
(x, Z) \in R_{S} \Leftrightarrow S(x) \cap Z \neq \emptyset .
$$

On the other hand, let $\langle X, \mathcal{T}, R\rangle$ be a normal monotonic $D S$-space. We define a relation $S_{R} \subseteq X \times X$ by

$$
(x, z) \in S_{R} \Leftrightarrow(x,(z]) \in R .
$$


Proposition 71. (1) Let $\langle X, \mathcal{T}, R\rangle$ be a normal monotonic DS-space. Then, the structure $\left\langle X, \mathcal{T}, S_{R}\right\rangle$ is a DS-space with a meet-relation $S_{R}$ such that $m_{R}(U)=$ $\mathrm{m}_{S_{R}}(U)$ for all $U \in \mathrm{Up}(X)$ and $R=R_{S_{R}}$.

(2) Let $\langle X, \mathcal{T}, S\rangle$ be a $D S$-space with a meet-relation $S \subseteq X \times X$. Then, the structure $\left\langle X, \mathcal{T}, R_{S}\right\rangle$ is a normal monotonic DS-space such that $m_{R_{S}}(U)=\mathrm{m}_{S}(U)$ for all $U \in \mathrm{Up}(X)$, and $S=S_{R_{S}}$.

Proof. 1. Let $U \in \mathrm{Up}(X)$. We will prove that $m_{R}(U)=\mathrm{m}_{S_{R}}(U)$. Let $x \in m_{R}(U)$ and $z \in S_{R}(x)$. Then, $(x,(z]) \in R$ and we get that $(z] \cap U \neq \emptyset$. Since $U$ is an upset, we have that $z \in U$. Thus, $S_{R}(x) \subseteq U$ and $x \in \mathrm{m}_{S_{R}}(U)$. Let $x \in \mathrm{m}_{S_{R}}(U)$ and $Z \in R(x)$. Then, there exists $z \in Z$ such that $(z] \in R(x)$. By definition $(x, z) \in S_{R}(x)$. So, $z \in U$ and we get that $Z \cap U \neq \emptyset$. Thus, $x \in m_{R}(U)$.

So, we have that $m_{R}(U)=\mathrm{m}_{S_{R}}(U) \in D(X)$ for all $U \in D(X)$. We will see that $S_{R}(x)=\bigcap\left\{U \in D(X): S_{R}(x) \subseteq U\right\}$ for all $x \in X$. Let $x, z \in X$ such that $z \in \bigcap\{U \in$ $\left.D(X): S_{R}(x) \subseteq U\right\}$. Then, $z \in U$ for all $U \in D(X)$ such that $x \in \mathrm{m}_{S_{R}}(U)=m_{R}(U)$. By condition (4) of Definition 26, $(z] \in R(x)$. Therefore, $z \in S_{R}(x)$.

Now, let $(x, Z) \in R$. We will see that $S_{R}(x) \cap Z \neq \emptyset$. Since $\langle X, \mathcal{T}, R\rangle$ is a normal monotonic $D S$-space, there exists $z \in Z$ such that $(z] \in R(x)$. By definition $z \in$ $S_{R}(x) \cap Z$. Let $(x, Z) \in R_{S_{R}}$. Then, $S_{R}(x) \cap Z \neq \emptyset$. Let $z \in Z$ such that $(x, z) \in S_{R}$. By definition of $S_{R},(x,(z]) \in R$. So, $(z] \subseteq Z$ and by Proposition $40,(x, Z) \in R$. Therefore $R=R_{S_{R}}$.

2. Let $U \in \operatorname{Up}(X)$. We will prove that $m_{R_{S}}(U)=\mathrm{m}_{S}(U)$. Let $x \in m_{R_{S}}(U)$ and $z \in S(x)$. Then, $S(x) \cap(z] \neq \emptyset$. By definition of $R_{S},(x,(z]) \in R_{S}$. So, $(z] \cap U \neq \emptyset$ and since $U$ is an upset, we have that $z \in U$. Thus, $S(x) \subseteq U$ and $x \in \mathrm{m}_{S}(U)$. Let $x \in \mathrm{m}_{S}(U)$ and let $Z \in R_{S}(x)$. Then, there exists $z \in Z$ such that $z \in S(x)$. So, $z \in U$ and we get that $Z \cap U \neq \emptyset$. Thus, $x \in m_{R_{S}}(U)$.

So, we have that $\mathrm{m}_{S}(U)=m_{R_{S}}(U) \in D(X)$ for all $U \in D(X)$. We will see that $\left\langle X, \mathcal{T}, R_{S}\right\rangle$ is a normal monotonic $D S$-space. Let $x \in X, Z \in \bigcap\left\{L_{U}: x \in m_{R_{S}}(U)\right\}$ and suppose that $Z \notin R_{S}(x)$. By definition, $Z \cap S(x)=\emptyset$ and since $S(x)$ is a closed subset, there exists $U \in D(X)$ such that $Z \subseteq U^{c}$ and $S(x) \cap U^{c}=\emptyset$, i.e., $x \in \mathrm{m}_{S}(U)=m_{R_{S}}(U)$ and $Z \cap U=\emptyset$, which is a contradiction. Therefore, $R_{S}(x)=\bigcap\left\{L_{U}: x \in m_{R_{S}}(U)\right\}$. Now, let $x \in X$ and $Z \in R_{S}(x)$. Then, there exists $z \in S(x) \cap Z$. So, $S(x) \cap(z] \neq \emptyset$ and by definition of $R_{S},(x,(z]) \in R_{S}$.

Finally, let $(x, z) \in S$. Then $z \in S(x) \cap(z]$ and, by definition, $(x,(z]) \in R_{S}$. Therefore, $(x, z) \in S_{R_{S}}$. On the other hand, let $(x, z) \in S_{R_{S}}$. Then, $(x,(z]) \in R_{S}$. So, $S(x) \cap(z] \neq \emptyset$. Since $S$ is a meet-relation, $S(x)$ is an upset. Thus, $z \in S(x)$. Therefore $S=S_{R_{S}}$.

Remark 72. Note that as a particular case we get the relation defined in [10] by Mai Gherke, where she gave an algebraic derivation of the space associated to a bounded distributive lattice with a modality $\square$ that preserves 1 and $\wedge$ based on the canonical extension. Given the modality $\square: A \rightarrow A$, since the extension $\square^{\sigma}=\square^{\pi}$ is completely meet preserving, it is completely determined by its action on the completely meet prime elements of the canonical extension. Working on Stone spaces, the family $\mathcal{S}(X(\mathbf{A}))$ is the family of all basic saturated sets and recall that $M^{\infty}(U p(X(\mathbf{A})))=\left\{\alpha\left(P^{c}\right)^{c}=(P]^{c}\right.$ : 
$P \in X(\mathbf{A})\}$. The relation $S \subseteq X(\mathbf{A}) \times X(\mathbf{A})$ defined in [10] is:

$$
(P, Q) \in S \Leftrightarrow \square^{\pi}\left((Q]^{c}\right) \subseteq(P]^{c} .
$$

It is easy to see that $\square^{\pi}\left((Q]^{c}\right) \subseteq(P]^{c} \Leftrightarrow \square^{-1}(P) \cap Q^{c}=\emptyset$.

5.3. Boolean Algebras with a monotonic operator. In paper [3] (see also [13] and [14]) S. Celani developed a topological duality between monotonic Boolean algebras and descriptive monotonic frames. These frames are actually monotonic $D S$-frames.

Recall that a Boolean algebra with a normal monotonic operation, is a pair $\langle\mathbf{A}, \square\rangle$ such that $\mathbf{A}$ is a Boolean algebra, and $\square$ is an operator defined on $A$ such that

(1) $\square(a \wedge b) \leq \square a \wedge \square b$ for all $a, b \in A$,

(2) $\square 1=1$.

Also, recall that a Stone space is a topological space $X=\langle X, \tau\rangle$ that is compact and totally disconnected, i.e., given distinct points $x, y \in X$, there exists a clopen (closed and open) subset $U$ of $X$ such that $x \in U$ and $y \notin U$. Let $\operatorname{Clop}(X)$ be the family of closed and open subsets of a Stone space $\langle X, \tau\rangle$.

A descriptive $m$-frame [3], or monotonic modal space, is a triple $\langle X, R, \tau\rangle$ such that

(1) $\langle X, \tau\rangle$ is a Stone space,

(2) $R \subseteq X \times \mathcal{C}_{0}(X)$, where $\mathcal{C}_{0}(X)=\mathcal{C}(X)-\{\emptyset\}$,

(3) $\square_{R}(U)=\{x \in X: \forall Y \in R(x)(Y \cap U \neq \emptyset)\} \in \operatorname{Clop}(X)$ for all $U \in \operatorname{Clop}(X)$,

(4) $R(x)=\bigcap\left\{L_{U}: x \in \square_{R}(U)\right\}$, for all $x \in X$.

Remark 73. It is well known that if $\langle X, \tau\rangle$ is a Stone space then $\operatorname{Clop}(X)$ is a basis for the topology and $\mathcal{S}(X)=\mathcal{C}(X)$. As $X$ is Hausdorff, the only irreducible closed sets are singletons, so $X$ is sober. Then, it is easy to see that any descriptive $m$-frame is a monotonic $D S$-space.

Remark 74. Let $\mathbf{A}=\langle A, \vee, \wedge, \neg, 0,1\rangle$ be Boolean algebra. Note that if $F$ is a filter of $\mathbf{A}$, then the set $I_{F}=\{\neg a: a \in F\}$ is an ideal of $\mathbf{A}$, and thus $\hat{F}=\alpha\left(I_{F}\right)$. Similarly, if $I$ is an ideal of $\mathbf{A}$, then the set $F_{I}=\{\neg a: a \in I\}$ is a filter of $\mathbf{A}$, and $\alpha(I)=\hat{F}_{I}$.

Let $\langle\mathbf{A}, \square\rangle$ be a Boolean algebra endowed with a normal monotonic operator $\square$. Let $\diamond: A \rightarrow A$ be the dual operator defined by $\diamond a=\neg \square \neg a$, for each $a \in A$. Following the construction of the $\mathcal{S}$ - and $\mathcal{C}$ - monotonic spaces, we have four relations: $G_{\diamond}, G_{\square}, R_{\diamond}$ and $R_{\square}$. The following proposition shows the relationships between them.

Proposition 75. Let $\langle\mathbf{A}, \square\rangle$ be a Boolean algebra endowed with a monotonic operator. Then, $G_{\diamond}=R_{\square}$ and $G_{\square}=R_{\diamond}$.

Proof. We will prove that $G_{\diamond}=R_{\square}$. Let $F \in \operatorname{Fi}(\mathbf{A})$ such that $(P, \hat{F}) \in G_{\diamond}$. Then, $F \subseteq \diamond^{-1}(P)$, i.e., for all $a \in F, \neg \square(\neg a) \in P$. So, $\square(\neg a) \notin P$, i.e., $\neg a \notin \square^{-1}(P)$. Thus, $\square^{-1}(P) \cap I_{F}=\emptyset$ and $\left(P, \alpha\left(I_{F}\right)\right) \in R_{\square}$. By remark, $\hat{F}=\alpha\left(I_{F}\right)$. The proof of other inclusion is similar.

The other equality is proved analogously. 


\section{REFERENCES}

[1] Calomino, I.M., Celani S.A.: Some remarks on distributive semilattices. Comment. Math. Univ. Carolin. 54 (3), 407-428 (2013) 1, 2, 2, 2.1, 4, 2.1, 4, 4.1

[2] Celani S.A.: Representation of Hilbert algebras and Implicative Semilattices. Central European Journal of Mathematics. 1 (4), 561--572 (2003) (document) 1

[3] Celani S.A.: Topological duality for boolean algebras with a normal n-ary monotonic operator. Order. 26 (1), 49-67 (2009) $1,5,5.3,5.3$

[4] Celani S.A.: Topological representation of distributive semilattices. Sci. Math. Japonicae Online. 8, 41-51 (2003) $1,2,2,2.1,2.1,4,4,4.1$

[5] Chagrov, A., Zakharyaschev, M.: Modal Logic. Volumen 35 of Oxford Logic Guides, New York (1997) 1

[6] Chajda, I., Halas, R., and Kühr, J.: Semilattice structures. Volumen 30, Research and Exposition in Mathematics, Heldermann Verlagr (2007) (document) 1, 2

[7] Chellas, B.F.: Modal logic: an introduction. Cambridge University Press (1980) 1,4

[8] Dunn, J.M., Gehrke, M., Palmigiano, A.: Canonical extensions and relational completeness of some substructural logics. J. Symbolic Logic. 70 (3), 713--740 (2005) 1, 2.2, 4, 4

[9] Düntsch, I., Orlowska, E., Rewitzky, I.: Structures with multirelations, their discrete dualities and applications. Fundamenta Informaticae. 100, 77-98 (2010) 4

[10] Gehrke, M.: Canonical extensions, Esakia spaces, and universal models. In: Bezhanishvili, G. (eds) Outstanding Contributions to Logic, volume 4, pp 9-41. Springer Netherlands (2014) 72

[11] Gehrke, M., Jónsson, B.: Monotone bounded distributive lattice expansions. Math Japonica. 52, $197-213$ (2000) $1,2.2$

[12] Grätzer, G.: General lattice theory. Academic Press. Birkhäuser Verlag (1978) 1, 2.1 , 2.1

[13] Hansen, H.H.: Monotonic modal logics. Master's thesis, University of Amsterdam, Faculty of Science, Institute for Logic, Language and Computation, (2003) $1,4,5.3$

[14] Hansen, H.H., Kupke, C., Pacuit, E.: Neighbourhood structures: Bisimilarity and basic model theory. Logical Methods in Computer Science, 5 (2), 1-38 (2009) 1, 5.3

[15] Jonsson, B., Tarski, A.: Boolean Algebras with Operators. Part I. American Journal of Mathematics. 73 (4), 891-939 (1951) 2.2

[16] Kojima, K.: Relational and neighborhood semantics for intuitionistic modal logic. Reports on Mathematical Logic. 47, 87-113 (2012) 1

[17] Rewitzky, I.: Binary multirelations. In: de Swart, H. , Orłowska, E., Schmidt, G., Roubens, M. (eds) Theory and Application of Relational Structures as Knowledge Instruments, volume 2929, pp. 259-274. Spinger-Verlag (2003) 4

[18] Sotirov, V.H.: Modal theories with intuitionistic logic. In Proceedings of the Conference on Mathematical Logic, Sofia, 1980. Bulgarian Academy of Sciences, Sofia, 139-171 (1984) 